\title{
Targeted delivery of immune therapeutics to lymph nodes prolongs cardiac allograft survival
}

\author{
Baharak Bahmani, ${ }^{1,2}$ Mayuko Uehara, ${ }^{1,2}$ Liwei Jiang, ${ }^{1,2}$ Farideh Ordikhani, ${ }^{1,2}$ Naima Banouni, ${ }^{1,2}$ Takaharu Ichimura, ${ }^{2}$ \\ Zhabiz Solhjou, ${ }^{1,2}$ Georg J. Furtmüller, ${ }^{3}$ Gerald Brandacher, ${ }^{3}$ David Alvarez, ${ }^{4}$ Ulrich H. von Andrian, ${ }^{4}$ Kenji Uchimura, ${ }^{5}$ \\ Qiaobing Xu, ${ }^{6}$ Ishaan Vohra, ${ }^{1,2}$ Osman A. Yilmam, ${ }^{1,2}$ Yousef Haik, ${ }^{7}$ Jamil Azzi,, ${ }^{1,2}$ Vivek Kasinath, ${ }^{1,2}$ Jonathan S. Bromberg, ${ }^{8}$ \\ Martina M. McGrath, ${ }^{1,2}$ and Reza Abdi ${ }^{1,2}$ \\ 'Transplantation Research Center and 'Renal Division, Brigham and Women's Hospital, Harvard Medical School, Boston, Massachusetts, USA. ${ }^{3}$ Department of Plastic and Reconstructive Surgery, Vascularized \\ Composite Allotransplantation Laboratory, Johns Hopkins University School of Medicine, Baltimore, Maryland, USA. ${ }^{4}$ Department of Microbiology and Immunobiology, Harvard Medical School, Boston, \\ Massachusetts, USA. ' Unite de Glycobiologie Structurale et Fonctionnelle, UMR 8576 CNRS, Universite de Lille 1, Villeneuve d’Ascq, France. ${ }^{6}$ Department of Biomedical Engineering, Tufts University, Medford, \\ Massachusetts, USA. 'ollege of Science and Engineering, Hamad bin Khalifa University, Doha, Qatar. ${ }^{8}$ Department of Surgery and Microbiology and Immunobiology, University of Maryland School of \\ Medicine, Baltimore, Maryland, USA.
}

\begin{abstract}
The targeted delivery of therapeutic drugs to lymph nodes (LNs) provides an unprecedented opportunity to improve the outcomes of transplantation and immune-mediated diseases. The high endothelial venule is a specialized segment of LN vasculature that uniquely expresses peripheral node addressin (PNAd) molecules. PNAd is recognized by MECA79 mAb. We previously generated a MECA79 mAb-coated microparticle (MP) that carries tacrolimus. Although this MP trafficked to LNs, it demonstrated limited therapeutic efficacy in our transplant model. Here, we have synthesized a nanoparticle (NP) as a carrier of anti-CD3, and optimized the conjugation strategy to coat the NP surface with MECA79 mAb (MECA79-antiCD3-NP) to enhance LN accumulation. As compared with nonconjugated NPs, a significantly higher quantity of MECA79NPs accumulated in the draining lymph node (DLN). Many MECA79-NPs underwent internalization by T cells and dendritic cells within the LNs. Short-term treatment of murine cardiac allograft recipients with MECA79-anti-CD3-NP resulted in significantly prolonged allograft survival in comparison with the control groups. Prolonged graft survival following treatment with MECA79-anti-CD3-NP was characterized by a significant increase in intragraft and DLN Treg populations. Treg depletion abrogated the prolongation of heart allograft survival. We believe this targeted approach of drug delivery could redefine the methods of administering immune therapeutics in transplantation.
\end{abstract}

\section{Introduction}

Organ transplantation has become a standard therapeutic strategy and has saved the lives of thousands of patients with irreversible end-stage organ failure. The key to its success has been the development of potent immunosuppressive agents (ISAs). However, the use of ISAs is associated with significant toxicity (1). Nanotechnology offers a unique opportunity to improve the therapeutic index of ISAs. A promising clinical application of nanotechnology is the targeted delivery of ISAs to the lymph nodes (LNs) following systemic administration, for more efficient suppression of alloimmunity (2). The rationale for devising this particular delivery platform arises from studies identifying the LN as a critical site of alloimmune regulation $(3,4)$. LNs are extremely compartmentalized secondary lymphoid organs that provide an optimal environment for freshly recruited lymphocytes to interact with antigen-presenting cells. The $\mathrm{LN}$ is a critical site for the priming and activation of alloreactive $\mathrm{T}$ cells, but it also plays an important

Authorship note: BB and MU contributed equally to this work. Conflict of interest: The authors have declared that no conflict of interest exists. License: Copyright 2018, American Society for Clinical Investigation. Submitted: March 8, 2018; Accepted: August 21, 2018 Reference information: J Clin Invest. 2018;128(11):4770-4786. https://doi.org/10.1172/JCl120923. role in the manipulation of the immune response toward regulation and tolerance $(2,5-7)$. The latter is highlighted by studies that detail the importance of the LN in promoting the activity of regulatory T cells (Tregs) $(8,9)$. Therefore, the ability to manipulate the microenvironment of LNs would provide a unique opportunity to control the direction of the immune response by directing it toward either a proinflammatory effector or an antiinflammatory tolerance response (10).

The vast majority of current LN-targeted delivery platforms rely on the administration of drugs to lymphatic vessels through the skin, but administration of therapeutics targeted to the LN systemically, via an intravascular route, expands their application to a broader spectrum of systemic diseases (11). The process of active targeting relies on the highly selective interaction of particle ligands with the site of interest, leading to intracellular drug release $(12,13)$. To ensure precise targeting, the surface of nanoparticles (NPs) can be coated with a number of materials (such as antibodies) to bind to a receptor of interest (12-16). In this manner, active nanodelivery significantly increases the drug payload at its specific target site (14-16). One of the key aspects of targeted delivery is the exclusiveness of the vasculature to which the NP localizes. The LN possesses a unique segment of vasculature known as the high endothelial venule (HEV), which exclusively expresses a family of sugar-coated membrane proteins referred to as peripheral 
node addressin (PNAd), which binds specifically to lymphocyteexpressed L-selectin (17). This binding initiates the rolling and tethering of lymphocytes, which precedes their migration through the HEV into the LN, where lymphocytes can encounter antigens and undergo activation. PNAd molecules are recognized by MECA79 monoclonal antibody (mAb) $(18,19)$. We have previously synthesized a MECA79 mAb-coated microparticle (MP) with the ability to carry tacrolimus and demonstrated the efficiency of this platform in targeting the draining lymph node (DLN), but many of these MPs became trapped inside the lungs. Reducing the size of a particle to nano-size $(100 \mathrm{~nm})$ decreases its entrapment in the lungs and thereby increases its circulatory time (20-22).

In this study, we have optimized our novel conjugation strategy to develop a nanoparticle (NP) coated with MECA79 mAb, which has the capacity to bind PNAd with improved efficiency, and also carries anti-CD3 as a payload (MECA79-anti-CD3-NP). The application of these substantial changes to our delivery platform was found to markedly enhance its efficacy in the prolongation of heart allograft survival.

\section{Results}

HEVs proliferate and expand in the DLN. The HEV is the target of our delivery platform, and as a proof of concept for its utility in this model, we compared the quantity and expansion of HEVs in a naive LN and a DLN following transplantation. A skin allograft (from $\mathrm{BALB} / \mathrm{c}$ ) was placed near the spine, behind the shoulder blade, of the recipient mice $(\mathrm{C} 57 \mathrm{BL} / 6)$, and allograft-draining axillary LNs (DLNs) were removed at day 8 after transplant. In comparison with the naive LN, the DLN was larger in size, and contained a higher quantity of HEVs (Supplemental Figure 1A, top panel; supplemental material available online with this article; https://doi.org/10.1172/ JCI120923DS1). Sections of naive LN and DLN were imaged by an EVOS Auto Imaging System to assess the number and size of the HEVs (bar graphs, ${ }^{* *} P<0.001, n=3$ mice per group; Supplemental Figure 1B). To visualize the HEV unit more precisely, the naive LN and DLN underwent 3D imaging of solvent-cleared organs (iDISCO), followed by MECA79 staining, which demonstrated a marked increase in the size of HEVs in the DLN, as compared with a naive LN (Supplemental Figure 1A, bottom panel). Blood flow is an important determinant of the delivery of particles to the DLN, and in keeping with HEV expansion, color Doppler echocardiography demonstrated much higher blood flow to the DLN, as compared with naive LN (Supplemental Figure 1C).

Synthesis and characterization of MECA79-IR800-NPs. To achieve targeted delivery to the LN, we have engineered NPs that bind to HEV-expressed PNAd and can mimic the footsteps of lymphocyte migration across the HEV. The PNAd molecules are a series of glycoproteins, coated with Sialyl-Lewis ${ }^{\mathrm{x}}$, and are bound by MECA79 mAb (18). We have previously demonstrated that MECA79-coated MPs accumulate in LNs, but we wished to ensure that NPs accumulate in a similar fashion. We therefore initially synthesized a PLGA-based NP loaded with IR800 dye (IR800-NP) via a single-step nanoprecipitation self-assembly method. MECA79 was then conjugated to the surface of the NP by pretreatment of MECA79 mAb with tris(2-carboxyethyl)phosphine hydrochloride (TCEP) to reduce the disulfide bonds and thereby create free thiol groups that could attach to maleimide groups on the surface of the
NP (Figure 1A). The hydrodynamic diameters of the nonconjugated (IR800-NP) and MECA79-conjugated (MECA79-IR800-NP) NPs were measured by dynamic light scattering as follows: IR800-NPs, $62.85 \pm 0.97 \mathrm{~nm}$; MECA79-IR800-NPs, $103.5 \pm 1.16 \mathrm{~nm}$. The surface charge of NPs was measured as $-16.2 \pm 0.67 \mathrm{mV}$ and $-8.86 \pm 0.43 \mathrm{mV}$, respectively ( ${ }^{* *} P<0.001, n=4-24$ per group) (Figure $\left.1 \mathrm{~B}\right)$. The NPs appeared uniformly spherical (Figure 1C).

Trafficking of MECA79-conjugated NPs to LNs. MECA79conjugated and nonconjugated NPs (each loaded with IR800 dye) were injected i.v. 7 days after transplantation of skin allografts. Live fluorescence imaging of the recipient performed at 24 hours after injection showed a higher fluorescent signal in the DLNs the axillary LNs (Axi LNs) and brachial LNs (Bra LNs) - of mice injected with MECA79-IR800-NPs, as compared with those injected with nonconjugated IR800-NPs (Figure 1D). The mean fluorescence intensity (MFI) of the DLN was significantly higher in MECA79-IR800-NP-injected mice than in the control group (noninjected) and IR800-NP-injected mice (control vs. IR800NP vs. MECA79-IR800-NP: 6,179 $\pm 2,307$ vs. $12,033 \pm 766.1$ vs. $33,779 \pm 3,096,{ }^{* * *} P<0.001, n=3$ mice per group, $1-2$ LNs from each mouse) (Figure 1E). We also examined the biodistribution of MECA79-conjugated and nonconjugated NPs into various organs following i.v. injection. No difference was noted in the trafficking of MECA79-conjugated and nonconjugated NP to the lung and spleen. However, a higher MFI was observed in the liver of animals treated with MECA79-IR800-NP, as compared with the control and IR800-NP (Figure 1E). MECA79-IR800-NPs localized to the DLNs at a much higher rate in comparison with the nondraining popliteal and para-aortic LNs (NDLNs), as measured by MFI (NDLN vs. DLN: $11,536 \pm 732.1$ vs. $33,264 \pm 2,046,{ }^{* * *} P<0.001$, $n=4-6$ mice per group, $2 \mathrm{LNs}$ from each mouse) (Figure $1 \mathrm{~F}$ ).

Next, we assessed whether NP egresses from the LN to the lymph fluid of the thoracic duct. We collected lymph from the thoracic duct at 1,3 , and 7 days following the injection of MECA79IR800-NPs ( $n=3$ mice). However, we were unable to detect MECA79-IR800-NPs in the lymph by fluorescence microscopy, indicating a lack of trafficking of NPs within the lymphatic system.

The accumulation of MECA79-NPs in LNs is dependent on its binding to PNAd-expressing HEV. Next, we examined the histological distribution of NPs within the DLN at 2, 6, and 24 hours after injection. Sections were stained for PNAd with MECA79 mAb (green), cell nuclei were stained with DAPI, and MECA79-NPs were visualized in red (Figure 2A). MECA79-NPs localized initially to the HEV (2 hours), then to the areas adjacent to the HEV (6 hours), and subsequently progressed into the interstitium of the DLN by 24 hours (Figure 2A). Significantly fewer NPs were detected in the DLNs of mice injected with nonconjugated NPs (Figure 2B). Given that MECA79 is an IgM antibody, we also used a nonspecific isotype IgM antibody as a control and tested the trafficking of IgM-conjugated NPs (IgM-NPs). Significantly fewer IgM-NPs trafficked to the LNs than MECA79-NPs, highlighting the specificity of MECA79 mAb in directing NPs effectively to the LNs (Supplemental Figure 2A).

We further tested the specificity of the trafficking of MECA79NPs through the use of a double-knockout (DKO) mouse, which lacks two $\mathrm{N}$-acetylglucosamine-6-O-sulfotransferase enzymes (GlcNAc6ST-1 and GlcNAc6ST-2) that are responsible for the 
A

IR800-NP

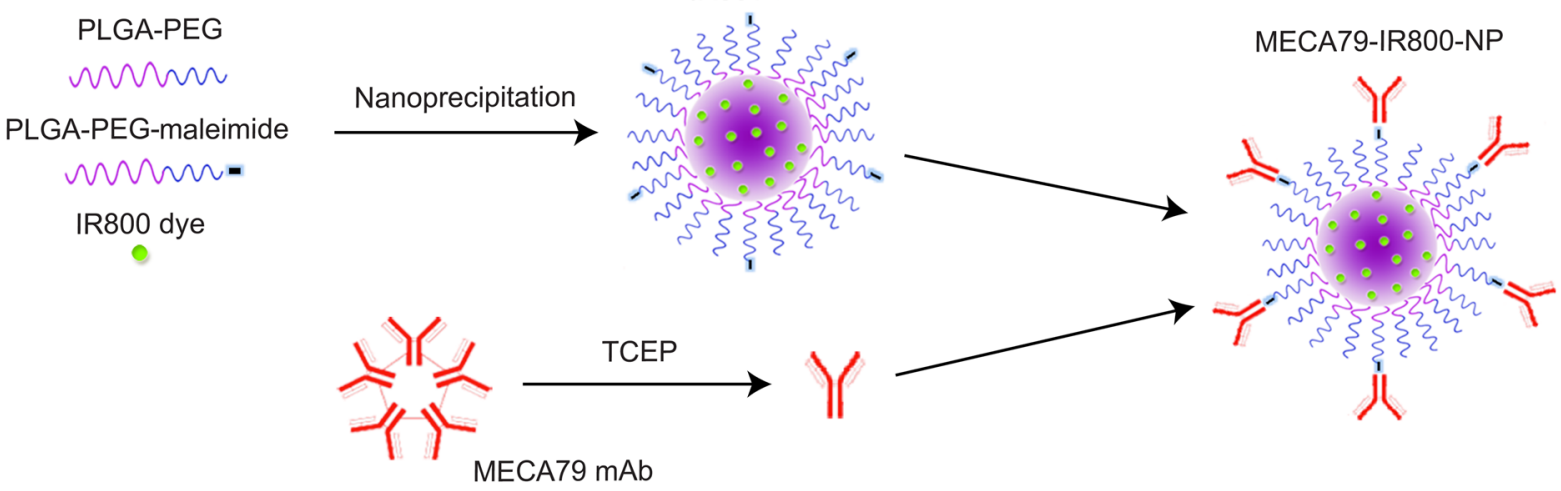

B
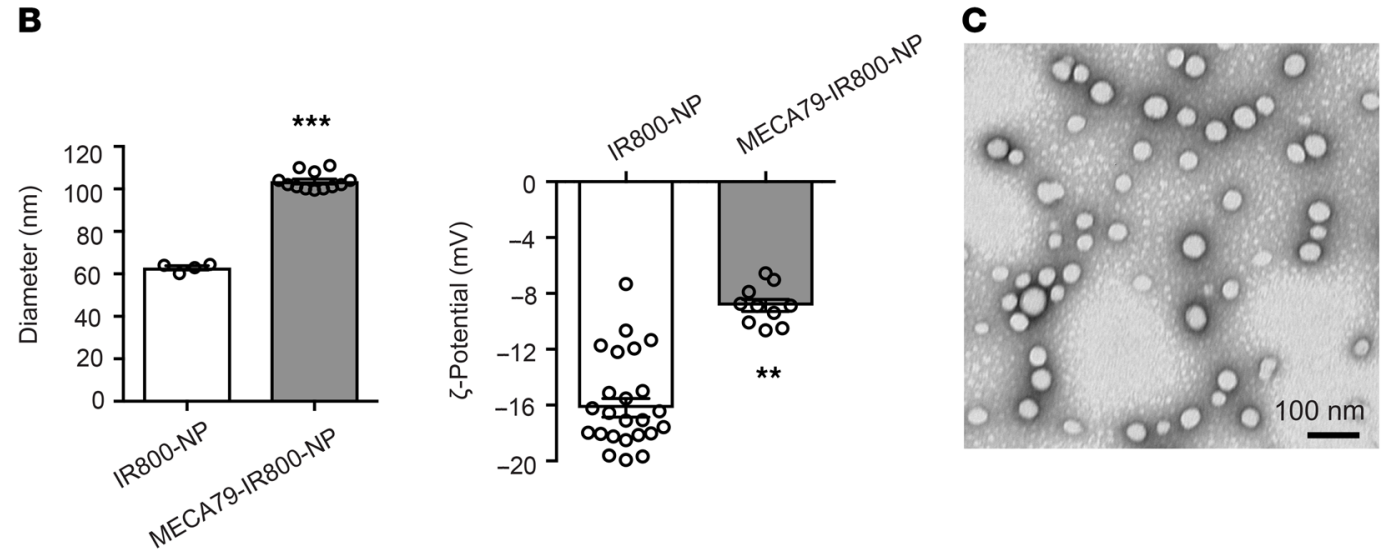

D

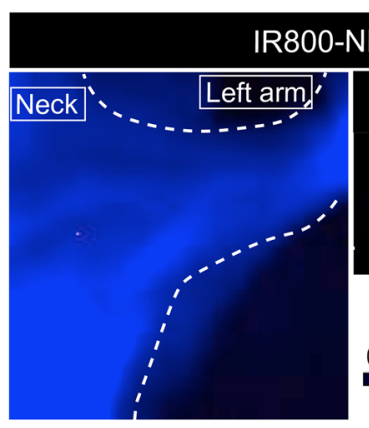

E

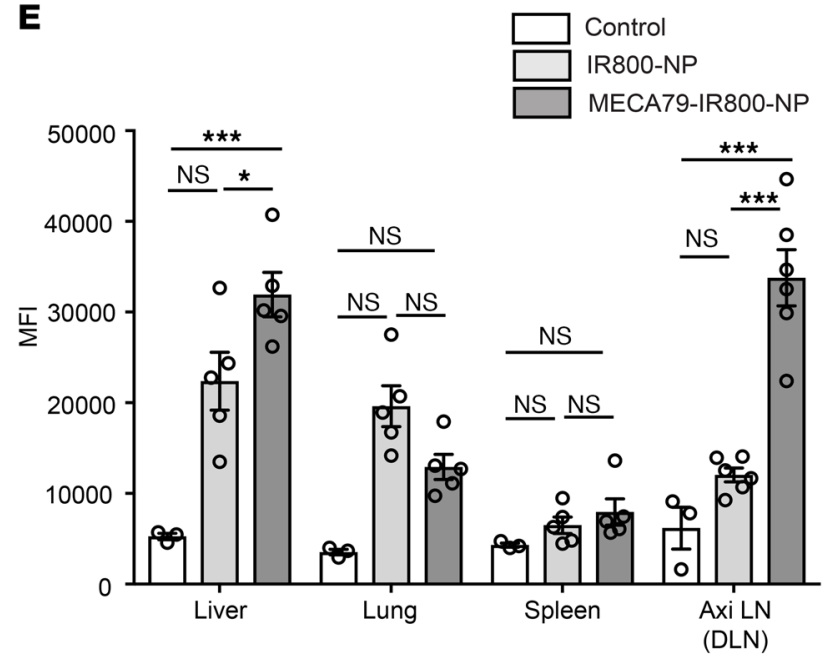

MECA79-IR800-NP

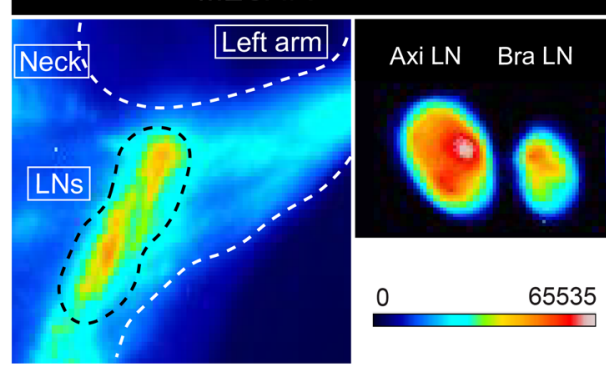

$\mathbf{F}$

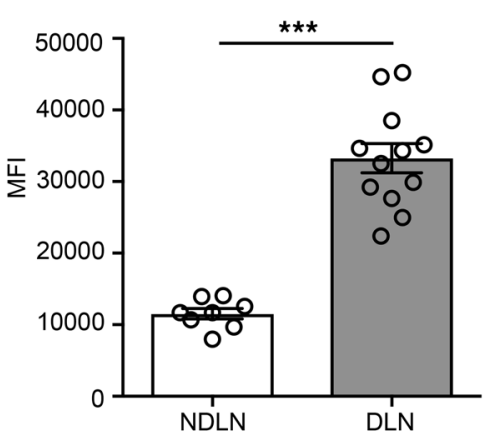


Figure 1. Targeted delivery of NPs to DLNs. (A) Schematic of IR800-NP synthesis and conjugation with MECA79 mAb. (B) Hydrodynamic size distribution and $\zeta$-potential of the IR800-NPs and MECA79-IR800-NPs (mean \pm SEM, Student's $t$ test, ${ }^{*} P<0.01,{ }^{* *} P<0.001$ ). (C) Representative transmission electron microscopy image of IR800-NPs. (D) Skin allograft recipients were injected i.v. with either IR800-NPs or MECA79IR800-NPs at day 7 after transplantation. Live fluorescence imaging at 24 hours after administration (i.v.) showed greater fluorescence signal in the DLNs (Axi LN and Bra LN) of mice injected with MECA79-IR800NPs. (E) Biodistribution of MECA79-conjugated and nonconjugated NPs, in addition to uninjected control, assessed via fluorescence imaging at 24 hours after injection (i.v.) of NPs (DLN: control vs. IR800-NP vs. MECA79-IR800-NP: 6,179 $\pm 2,307$ vs. $12,033 \pm 766.1$ vs. 33,779 $\pm 3,096$, mean $\pm \mathrm{SEM}$, ANOVA test, ${ }^{*} P<0.05,{ }^{* *} P<0.001, n=3$ mice per group, 1-2 LNs from each mouse). (F) Trafficking of MECA79-conjugated NPs into DLNs and NDLNs (NDLN vs. DLN: $11,536 \pm 732.1$ vs. 33,264 $\pm 2,046$, mean \pm SEM, Student's $t$ test, ${ }^{* *} P<0.001, n=4-6$ mice per group, 2 LNs from each mouse).

biosynthesis of L-selectin ligands in the HEV (23). Twenty-four hours after the injection of MECA79-IR800-NPs into naive DKO mice, their axillary LNs were harvested and stained for PNAd and CD31. No PNAd expression or MECA79-IR800-NPs were detected in the LNs of the DKO mice (Figure 2C). Sialyl-Lewis ${ }^{\mathrm{X}}$, a sugar moiety of PNAd, is also expressed on lymphocytes (17). We injected MECA79-NPs into NOD-scid mice, in order to exclude the possibility that NPs accumulate in the LNs by binding to lymphocytes that shuttle them into the LNs. Imaging performed at 24 hours after injection revealed that the MECA79-NPs accumulated to a similar extent in the LNs of NOD-scid mice versus WT mice $(34,356 \pm 837.7$ vs. $31,918 \pm 2,515, P=\mathrm{NS}, n=3$ mice per group, 2 LNs from each mouse), indicating that this process is independent of the presence of lymphocytes (Figure 2D).

Next, we examined whether leukocytes in the LN internalized NPs. At 24 hours after injection, LNs were harvested and stained for CD11c, CD11b, B220, and CD3. The majority of MECA79-NPs costained with CD11c and CD3 (Figure 2E). To further examine the capacity of $\mathrm{CD} 11 \mathrm{c}^{+}$dendritic cells (DCs) to internalize our NPs, we isolated and cultured murine DCs in vitro with IR800NPs, which confirmed that these NPs are internalized by DCs (Supplemental Figure 2B).

Characterization and optimization of the conjugation of MECA79 $m A b$ to NPs. The process of antibody conjugation often creates heterogeneous antibody products $(24,25)$. We used a native (nondenaturing) gel to assess the molecular weight (MW) of the antibody products formed following the reduction of MECA79 mAb with TCEP. Intact MECA79 mAb appears to be a mixture of a pentamer and a hexamer (Figure 3A). Decreasing the ratio of MECA79 mAb to TCEP created by-products with MWs of approximately 400 and $200 \mathrm{kDa}$, and maximizing this ratio created a monomer with MW of approximately $200 \mathrm{kDa}$ (Figure 3A). Then, we tested the efficacy of each conjugation protocol by examining the effect of the various antibody by-products on the in vivo trafficking of the NPs. The MFI of LNs isolated from animals 24 hours after i.v. injection of NPs was compared and is shown in Figure 3B. Reduction of MECA79 mAb into a monomer-only product of approximately $200 \mathrm{MW}$ resulted in the most robust trafficking of NPs to the LNs (Figure 3B).

Development of immunotherapeutic MECA79-NPs for the effective suppression of transplant rejection. Short-course induction therapy using highly potent and selective agents in the initial phase after transplantation has reduced the incidence of acute rejection $(26,27)$ and emerged as the standard of care for transplant patients $(28,29)$. Anti-CD3 mAbs are some of the most wellstudied immunotherapeutic agents used in transplantation and autoimmune diseases $(30,31)$. Anti-CD3 mAbs promote Tregs but suppress pathogenic T cells (32-34).

We encapsulated anti-CD3 mAb in NPs (anti-CD3-NPs) using a double-emulsion/solvent evaporation method. The conjugation of MECA79 mAb to the surface of NPs (MECA79-anti-CD3-NPs) was carried out using the optimal reduction protocol described above. The average diameter of NPs was $80.9 \pm 2.88 \mathrm{~nm}$ before MECA79 mAb conjugation and $123.7 \pm 8.3 \mathrm{~nm}$ after conjugation, as determined by dynamic light scattering $\left({ }^{* *} P<0.01, n=3\right.$ per group) (Figure 3C). The loading efficiency for anti-CD3 was $30 \%$ $\pm 5 \%$. We validated the controlled release of anti-CD3 mAb from NPs, by studying the release profile of anti-CD3 for 2 weeks in vitro. The results confirmed the gradual release profile of antiCD3 over time (Figure 3D).

The use of organic solvents during the fabrication of NPs may affect the function of the antibodies. We therefore next tested the activity of anti-CD3 mAb after encapsulation, by testing its ability to stimulate $\mathrm{T}$ cell proliferation in an in vitro assay. A similar level of $\mathrm{T}$ cell proliferation was observed in both free anti-CD3-treated and anti-CD3-NP-treated cells (percentage $\mathrm{T}$ cells proliferating: free anti-CD3 and anti-CD3-NP vs. negative control, ${ }^{* * *} P<0.01$, $n=3$ mice per group) (Supplemental Figure $3 \mathrm{~A}$ ).

Importantly, we were interested in the specific delivery of anti-CD3 mAb to the DLN. Skin-transplanted mice were injected with 1 dose of MECA79-anti-CD3-NPs (5 $\mu \mathrm{g}$ of anti-CD3) versus free anti-CD3 (5 $\mu$ g of anti-CD3) at 7 days after transplantation. The DLNs from treated mice were removed 24 hours later. Protein was extracted and analyzed by Western blot, and these data were compared with data from DLNs from uninjected control mice (Figure 3E). Markedly higher levels of anti-CD3 were detected in the DLNs of mice treated with MECA79anti-CD3-NP as compared with the free anti-CD3 and control groups, respectively (control vs. free anti-CD3 vs. MECA79anti-CD3-NP: 0 vs. $0.87 \pm 0.09$ vs. $1.43 \pm 0.14,{ }^{* *} P<0.001, n=4$ mice per group) (Figure $3 \mathrm{E}$ ).

To elaborate further on the cellular delivery of anti-CD3, we synthesized an NP carrying Alexa Fluor 488-labeled (AF488-labeled) anti-CD3 (*anti-CD3). We then injected skin-transplanted mice with either MECA79-*anti-CD3-NPs or *anti-CD3-NPs. The DLNs from these mice were assessed for the percentage of fluorescently labeled $\mathrm{T}$ cells 24 hours after injection. We observed a significantly greater number of fluorescently labeled $\mathrm{CD} 4^{+}, \mathrm{CD}^{+}$, and $\mathrm{CD} 4^{+} \mathrm{CD} 69^{+}$ $\mathrm{T}$ cells, Tregs, and CD11 $\mathrm{c}^{+}$cells in the DLNs of mice treated with MECA79-*anti-CD3-NP as compared with *anti-CD3-NP (Figure $3 F)$. In contrast, there was no difference between the 2 groups in the frequency of fluorescently labeled B cells in the DLNs (Figure 3F).

DCs could also potentially release *anti-CD3 following the uptake of NPs. To assess this, we incubated isolated splenic DCs with either ${ }^{*}$ anti-CD3 or ${ }^{*}$ anti-CD3-NPs for 1 hour. DCs were washed and then cultured with T cells for 6 hours, and then $\mathrm{T}$ cells were analyzed by flow cytometry to assess for AF488labeled T cells. A significantly higher percentage of labeled CD $4^{+}$ 
A

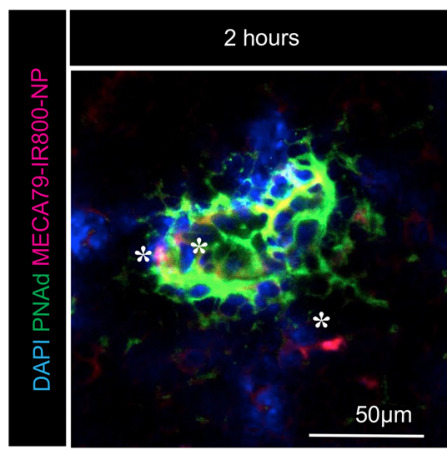

C

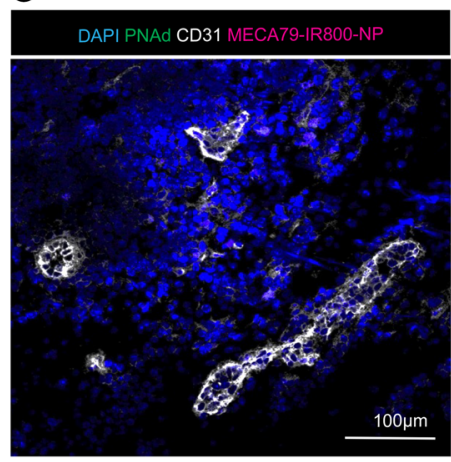

$\mathbf{E}$
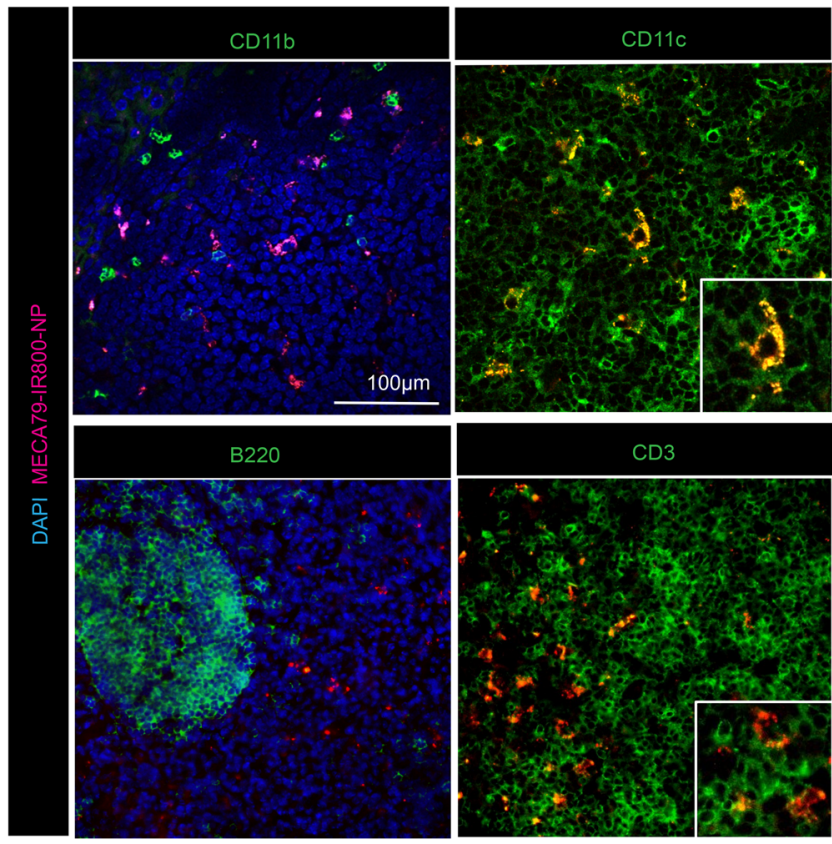

and $\mathrm{CD} 8^{+} \mathrm{T}$ cells were observed in the group incubated with DCs precultured with *anti-CD3-NPs compared with those incubated with ${ }^{*}$ anti-CD3 ( ${ }^{*}$ anti-CD3 vs. ${ }^{*}$ anti-CD3-NP: $2.08 \% \pm 0.35 \%$ vs. $39.5 \% \pm 2.01 \%$ for $\mathrm{CD}^{+}, 2.52 \% \pm 0.23 \%$ vs. $26.2 \% \pm 1.97 \%$ for $\mathrm{CD}^{+},{ }^{* * * *} \mathrm{P}<0.0001, n=4$ per group) (Figure $3 \mathrm{G}$ ), suggesting that DC uptake and release of anti-CD3 may occur more frequently after exposure to anti-CD3-loaded NPs.

Finally, we examined the effect of free anti-CD3 and MECA79anti-CD3-NPs on the cytokine profile of donor-reactive $\mathrm{T}$ cells
B

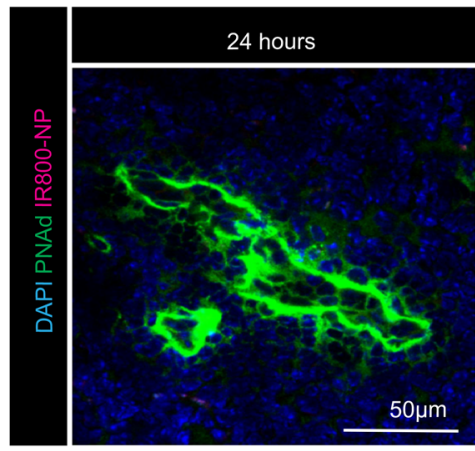

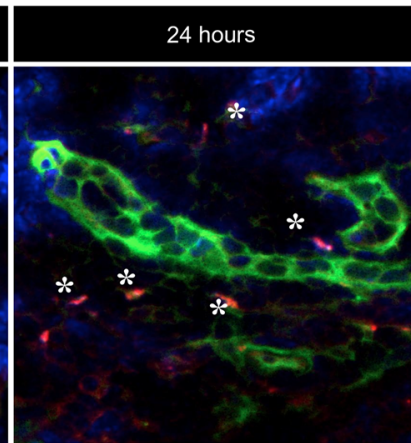

NS

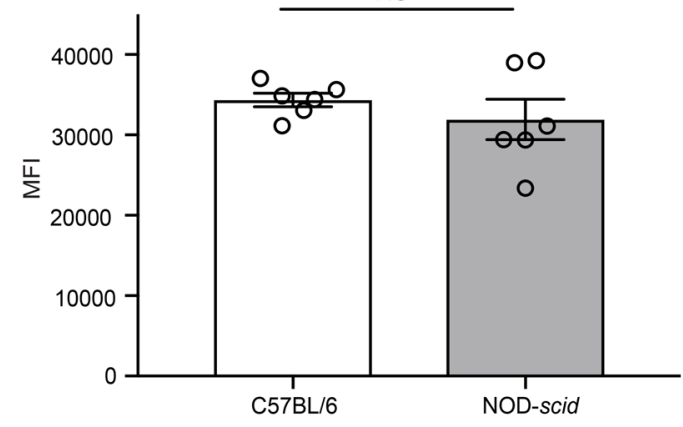

Figure 2. Histological characterization of MECA79-NP trafficking in the DLN. (A) MECA79-IR800-NPs (in red) can be detected in the HEV (in green) at early time points and infiltrate to adjacent areas in parenchyma of LNs at later time points. (B) Nontargeted IR800-NPs were not detected in the DLN. (C) Lack of PNAd expression and impaired trafficking of MECA79IR800-NPs to the LNs of double-knockout mice. (D) MFI quantification of axillary LNs showing no difference between $\mathrm{C} 57 \mathrm{BL} / 6$ mice and NOD-scid mice at 24 hours after injection of MECA79-IR800-NPs (34,356 \pm 837.7 vs. $31,918 \pm 2,515$, mean \pm SEM, Student's $t$ test, $P=N S, n=3$ mice per group, 2 LNs from each mouse). (E) Immunofluorescence analysis of the DLN showed colocalization of MECA79-IR800-NPs with $\mathrm{CD}_{11 \mathrm{C}^{+}}$and $\mathrm{CD}^{+}$cells (scale bar: $100 \mu \mathrm{m}$ ).

within the LNs. DLNs were harvested from recipient mice on day 7 after skin transplant, and isolated $\mathrm{T}$ cells were stimulated with irradiated donor cells. Cytokine levels were measured by Luminex assay (MilliporeSigma) of culture supernatant at 48 hours. Treatment with free anti-CD3 and MECA79-anti-CD3-NP led to marked and similar suppression of IFN- $\gamma$, TNF- $\alpha$, and IL- 6 production in comparison with untreated controls (Supplemental Figure 3B).

Treatment with MECA79-anti-CD3-NP prolongs heart allograft survival. Next, we examined the efficacy of MECA79-anti-CD3- 
NPs in the suppression of transplant rejection using a murine heart transplant model. BALB/c hearts were transplanted into C57BL/6 recipient mice. First, we tested the therapeutic efficacy of 2 different conjugation conditions for the synthesis of MECA79anti-CD3-NP - MECA79/TCEP ratios of 10:2 and 30:30 - with respect to the postponement of transplant rejection. To compare the efficacy of these NPs in preventing rejection, cardiac allograft recipient mice were injected i.v. with MECA79-anti-CD3-NPs on days $-1,0,1,2$, and 3 ( $5 \mu$ g of anti-CD3 per day) following transplantation. Consistent with the trafficking data (see Figure 3B), heart graft survival was significantly longer following MECA79 mAb conjugation using the $30: 30$ ratio as opposed to the $10: 2$ ratio (mean survival time [MST] 117.5 days vs. 22 days, respectively, ${ }^{* *} P$ $<0.01, n=3-5$ mice per group) .

Having identified the optimal formulation for MECA79-antiCD3-NPs, we then investigated their efficacy in prolonging heart allograft survival, when compared with other forms of delivery of anti-CD3. Mice with transplants were treated with unconjugated NP, MECA79-NP, free anti-CD3, anti-CD3-NP, or MECA79anti-CD3-NP from days -1 to 3 post-transplantation (all anti-CD3 dosing was $5 \mu \mathrm{g} / \mathrm{d})$. We observed a highly significant prolongation of cardiac transplant survival following treatment with MECA79anti-CD3-NP (MST of untreated control recipient, NP-alone, MECA79-NP, free anti-CD3, anti-CD3-NP, and MECA79-antiCD3-NP heart allografts was 7, 7, 7, 30, 35, and 117.5 days, respectively, $n=5-9$ mice per group) (Figure $4 \mathrm{~A}$ ). These data indicate that targeted delivery of anti-CD3 to LNs dramatically increases its efficacy in inducing long-term allograft survival.

We next sought to confirm the specific role of MECA79 binding to PNAd in targeted anti-CD3 delivery, and the greater efficacy of MECA79-anti-CD3-NPs. We generated NPs loaded with anti-CD3, and then conjugated with a control isotype $\operatorname{IgM}$ antibody. Heart allograft survival following treatment with IgM-antiCD3-NP was comparable to that the groups treated with free antiCD3 or non-conjugated anti-CD3-NP (MST 37 vs. 30 vs. 35 days, respectively, $P=$ NS, $n=4-9$ mice per group; Figure $4 \mathrm{~A})$. This indicates the essential role of MECA79 conjugation in MECA79-antiCD3-NPs to target anti-CD3 to LNs and maximize its efficacy.

We next sought to clarify the importance of various secondary lymphoid tissues as sites of action of MECA79-anti-CD3-NPs. $\mathrm{BALB} / \mathrm{c}$ heart allografts were transplanted into lymphotoxin$\alpha$-knockout recipients (LT $\alpha-\mathrm{KO}, \mathrm{C} 57 \mathrm{BL} / 6$ background), which lack LNs. We observed markedly decreased allograft survival in $\mathrm{LT} \alpha-\mathrm{KO}$ recipients as compared with WT following treatment with MECA79-anti-CD3-NP (MST of 45 days vs. 117.5 days, $n=$ 5 mice per group). We next transplanted fully mismatched cardiac allografts into splenectomized C57BL/6 mice. The lack of a spleen did not alter transplant survival following treatment with MECA79-anti-CD3-NP (MST $>100$ days, $n=5$ mice per group). These data indicate that the capacity of MECA79-anti-CD3-NPs to prolong cardiac transplant survival is dependent on the presence of LNs, but independent of the spleen.

We next assessed the importance of timing of administration of NPs for maximal therapeutic efficacy. We assessed 3 alternative dosing regimens: treatment of transplant recipients on day -1 alone, on days $0-3$, or on days 3-5 post-transplant. Compared with our standard dosing regimen, there was little therapeutic effect, with MSTs of 12,18 , and 12 days, respectively ( $n=3-5$ mice per group). These data indicate that maximal benefit is achieved with dosing beginning prior to transplantation on day -1 and continuing through to day 3 to prevent alloimmune activation within the DLN.

Despite long-term survival, allografts can sustain significant subclinical immune-mediated damage. To assess this, BALB/c cardiac allografts were transplanted into $\mathrm{C} 57 \mathrm{BL} / 6$ recipients, treated with free anti-CD3 versus MECA79-anti-CD3-NP on day -1 to day 3 post-transplant ( $5 \mu \mathrm{g}$ of anti-CD3 per mouse each day), and harvested at day 40 after transplant. Hearts were examined histologically, using a score modified from the International Society for Heart and Lung Transplantation $(35,36)$. Grafts from recipients treated with free anti-CD3 showed moderate cellular infiltration in myocytes and perivascular regions, thickened vascular walls, and occluded arteries. However, those treated with MECA79-anti-CD3NP showed significantly less cellular infiltration, with healthy myocytes and lower vascular injury score (free anti-CD3 vs. MECA79anti-CD3-NP: $2.209 \pm 0.34$ vs. $0.979 \pm 0.31$ for cellular infiltration score, respectively, ${ }^{*} P<0.05 ; 3.954 \pm 0.67$ vs. $1.738 \pm 0.38$ for vascular score, respectively, ${ }^{*} P<0.05 ; n=4$ mice per group) (Figure $4 \mathrm{~B}$ ). On immunofluorescence staining, fewer $\mathrm{T}$ cells and macrophages were present in the heart allografts treated with MECA79-anti-CD3$\mathrm{NP}$, as compared with free anti-CD3 (Figure 4C). We also examined long-term surviving heart allografts of MECA79-anti-CD3-NPtreated mice at the time of rejection (125 days after transplantation). Heart allografts showed myocyte necrosis, fibrosis, and cellular infiltrates (Supplemental Figure 3C), indicating that treatment with NP at the time of transplant is insufficient to control long-term alloimmune responses.

Mechanisms of suppression of alloimmune responses by MECA79anti-CD3-NP. We next assessed the effect of treatment with MECA79-anti-CD3-NP on the T cell response in the DLN and allograft. C57BL/6 recipients of BALB/c hearts were treated with free anti-CD3 or MECA79-anti-CD3-NP, and on day 17, DLNs (paraaortic LNs) and hearts were harvested for analysis by flow cytometry.

Overall, $\mathrm{CD}^{+} \mathrm{T}$ cells were not depleted significantly in the DLN, and we did not observe any significant impact on the T cell repertoire (TCR V $\alpha 2, \beta$, and $\gamma / \sigma$ chains) in the DLNs from mice treated with either free anti-CD3 or MECA79-anti-CD3-NP (data not shown). Furthermore, no significant differences were observed in the percentages of $\mathrm{CD} 4{ }^{+} \mathrm{CD} 44^{\text {hi }} \mathrm{CD} 62 \mathrm{~L}^{\text {lo }} \mathrm{T}$ cells, Tregs, and IFN- $\gamma$-producing CD4 ${ }^{+} \mathrm{T}$ cells in DLNs of the 2 treatment groups (free anti-CD3 vs. MECA79-anti-CD3-NP: $14.6 \%$ $\pm 2.6 \%$ vs. $15.6 \% \pm 1.8 \%, P=\mathrm{NS}$, for $\mathrm{CD} 4{ }^{+} \mathrm{CD} 444^{\text {hi }} \mathrm{CD} 62 \mathrm{~L}^{\text {lo }}$ cells; $12.4 \% \pm 1.9 \%$ vs. $12.1 \% \pm 1.1 \%, P=$ NS, for Tregs; $4.5 \% \pm 0.9 \%$ vs. $3.2 \% \pm 0.3 \%, P=\mathrm{NS}$, for IFN- $\gamma$-producing $\mathrm{CD}^{+}{ }^{+}$cells; $n=4$ mice per group) (Supplemental Figure 4A). The lack of observed effect of either treatment on T cell population within the DLN could relate to the timing of $\mathrm{LN}$ harvest in relation to treatment.

Given the lack of changes in DLN T cell populations to explain the observed prolonged graft survival, we next phenotyped the intragraft $\mathrm{T}$ cell populations by flow cytometry. We noted a marked reduction in the total number of graft-infiltrating $\mathrm{CD}^{+}$cells following treatment with MECA79-anti-CD3-NP (free anti-CD3 vs. MECA79-anti-CD3-NP: $4.761 \times 10^{6} \pm 0.9 \times 10^{6}$ vs. $1.728 \times 10^{6} \pm 0.9$ $\times 10^{6},{ }^{*} P<0.05, n=4$ mice per group) (Figure $5 \mathrm{~A}$ ). We then ana- 
A

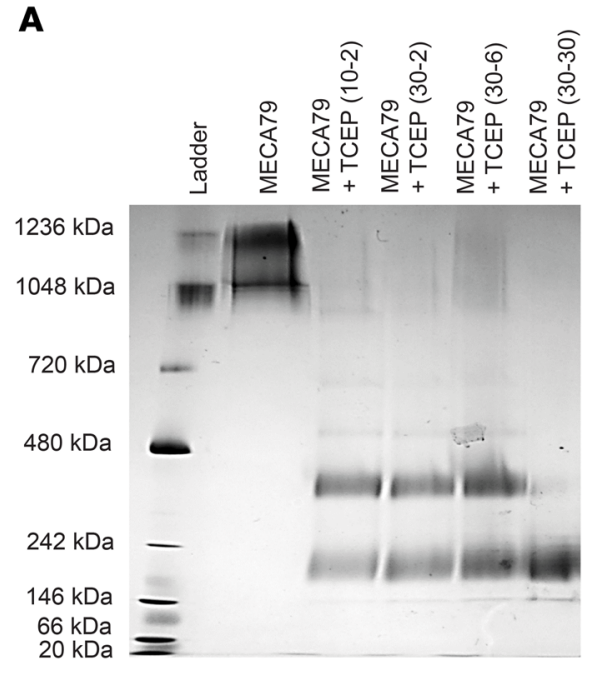

C

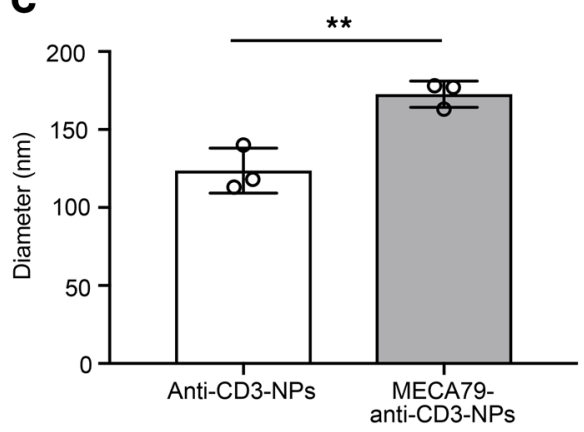

B

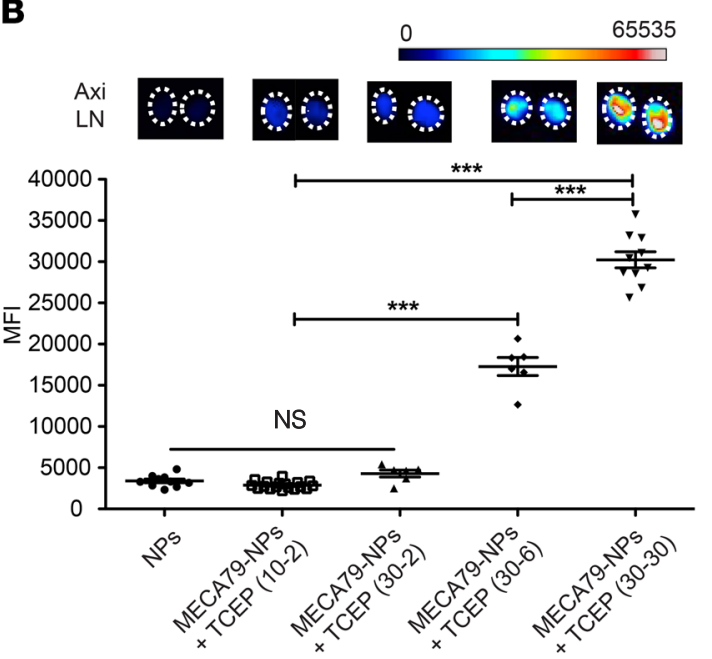

D

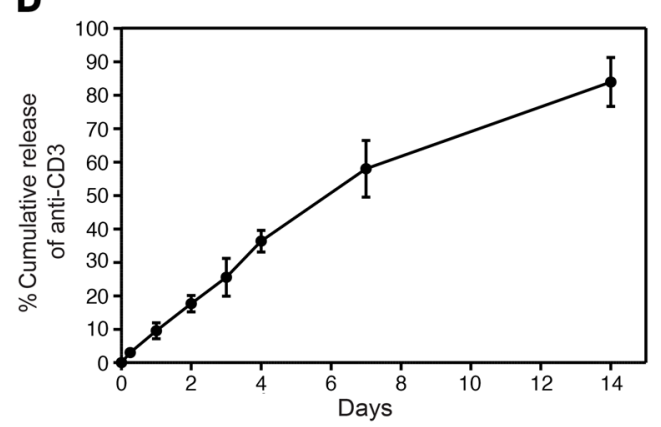

E

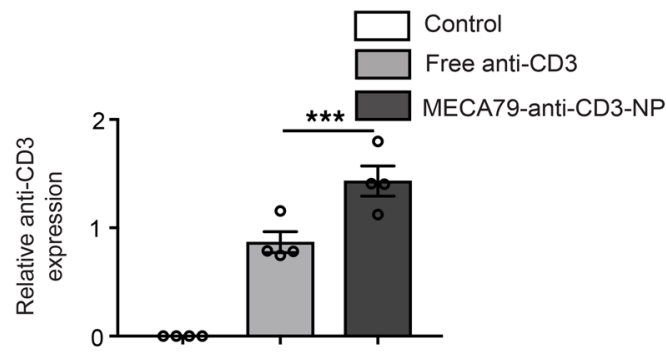

$\mathbf{F}$
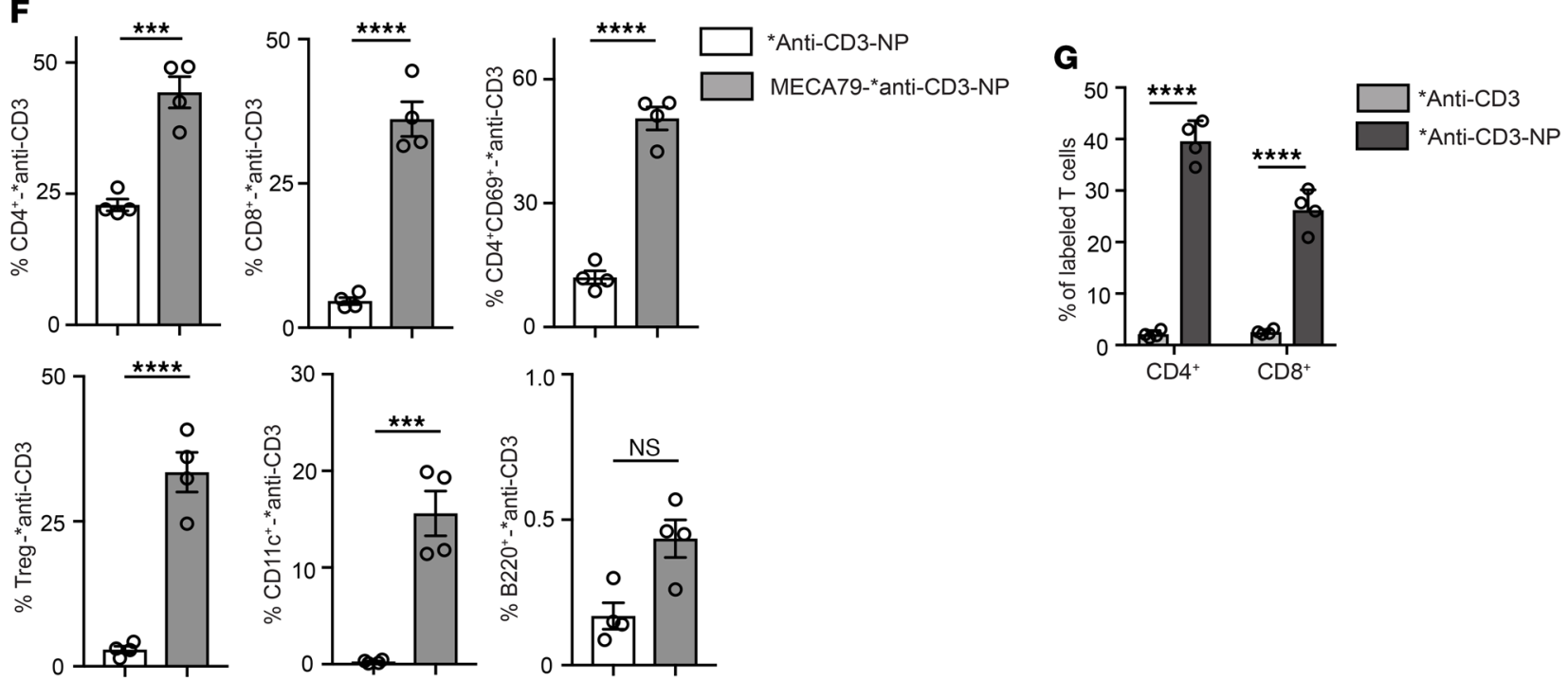
Figure 3. Optimization of conjugation of MECA79 mAb to the NPs. (A) Native (nonreducing) 3\%-12\% gel showed the effect of the reduction of MECA79 mAb with various MECA79/TCEP ratios on its molecular weight. (B) Corresponding trafficking of MECA79-NPs to LNs, as assessed by fluorescence imaging (mean \pm SEM, Student's $t$ test, ${ }^{* *} P<0.001, n=3-5$ mice per group, 2-4 LNs from each mouse). (C) Hydrodynamic diameter of anti-CD3NPs versus MECA79-anti-CD3-NPs (mean \pm SEM, Student's $t$ test, ${ }^{*} P<$ $0.01, n=3$ per group). (D) Sustained release profile of anti-CD3 from NPs over a duration of 14 days ( $n=3$ per group). (E) Western blot analysis of DLNs showed a significant increase in anti-CD3 expression in the MECA79-antiCD3-NP-treated group ( $5 \mu \mathrm{g}$ of anti-CD3) in comparison with the no-treatment and free anti-CD3 groups ( $5 \mu \mathrm{g}$ of anti-CD3). $\beta$-Actin was used as a loading control. The fold change over the syngeneic sample was calculated after densitometric analysis and correction for $\beta$ actin (control vs. free antiCD3 vs. MECA79-anti-CD3-NP: 0 vs. $0.87 \pm 0.09$ vs. $1.43 \pm 0.14$, mean \pm SEM, Student's $t$ test, ${ }^{* * *} P<0.001, n=4$ mice per group). (F) *anti-CD3-NPs with or without MECA79 antibody conjugation were injected into skin-transplanted mice. The DLNs from MECA79-*anti-CD3-NP-treated mice contained a significantly greater percentage of *anti-CD3-labeled T cells and DCs than controls. No differences were seen in *anti-CD3-labeled B cells (mean \pm SEM, Student's $t$ test, ${ }^{* * *} P<0.001,{ }^{* * *} P<0.0001, n=4$ mice per group). (C) DCs were cultured in vitro with *anti-CD3 or *anti-CD3-NPs, washed, and reincubated with $\mathrm{CD}^{+}$and $\mathrm{CD} 8^{+} \mathrm{T}$ cells, and the degree of $\mathrm{T}$ cell labeling was analyzed. Significantly greater labeling with *anti-CD3 was observed in both $\mathrm{CD} 4^{+}$and CD8 ${ }^{+} \mathrm{T}$ cells incubated with *anti-CD3 NP cultured DCs as compared with those cultured with *anti-CD3 alone (*anti-CD3 vs. *anti-CD3-NP: $2.08 \pm$ 0.35 vs. $39.5 \pm 2.01$ for $\mathrm{CD}^{+}, 2.52 \pm 0.23$ vs. $26.2 \pm 1.97$ for $\mathrm{CD}^{+}$, mean $\pm \mathrm{SEM}$, Student's $t$ test, ${ }^{* * * *} P<0.0001, n=4$ per group).

lyzed the $\mathrm{T}$ cell infiltrate, and observed a more pro-tolerogenic $\mathrm{T}$ cell profile in MECA79-anti-CD3-NP-treated recipients. While the absolute number of intragraft Tregs was similar (data not shown), the percentage of intragraft Tregs was significantly higher after treatment with MECA79-anti-CD3-NP as compared with free anti$\mathrm{CD} 3\left(21.38 \% \pm 2.65 \%\right.$ vs. $13.23 \% \pm 2.10 \%,{ }^{*} P<0.05, n=4$ mice per group) (Figure $5 \mathrm{~A})$. Similarly, the ratio of CD8 effector T cells $\left(\mathrm{CD} 44^{\text {hi }} \mathrm{CD} 62 \mathrm{~L}^{\mathrm{lo}}\right)$ to Tregs was significantly lower in MECA79-antiCD3-NP-treated grafts as compared with the free anti-CD3 group (Figure 5A), indicating a shift toward a more Treg-dense infiltrate.

Since we observed a significant increase in the percentage of intragraft Tregs in MECA79-anti-CD3-NP-treated mice, we investigated the importance of Tregs in long-term allograft survival. C57BL/6 mice underwent transplantation, were treated with MECA79-anti-CD3-NP, and underwent Treg depletion by i.v. injection of anti-CD25 antibody (200 $\mu$ i.v. per mouse) at 25 days after transplantation. Treatment with anti-CD25 antibody led to accelerated rejection (MST of 59 days after transplant [34 days after anti-CD25 injection] vs. 117.5 days in non-Treg-depleted controls, $n=5$ mice per group), which suggests that Tregs may play an important role in prolongation of heart graft survival following treatment with MECA79-anti-CD3-NP.

To dissect the process of immune activation and regulation in the LNs in greater detail, we then used an OVA-based mouse model of transplantation, in which immune activation in the DLN occurs during a narrower window of time. Hearts from OVAtransgenic mice were transplanted into $\mathrm{Rag}^{-/}$mice into which $\mathrm{CD} 4^{+}$. OTII T cells and CD8+.OTI T cells had been adoptively transferred. The mice received no treatment, free anti-CD3, or MECA79anti-CD3-NP and were sacrificed on day 5 after transplant, when DLNs and heart were harvested. The DLNs of the MECA79-anti-
CD3-NP-treated group contained a significantly higher proportion of Tregs $(61.7 \% \pm 4.9 \%)$ in comparison with untreated $(27.9 \% \pm$ $4.8 \%)$ and free anti-CD3 treatment groups $\left(36.2 \% \pm 9.7 \%,{ }^{*} P<0.05\right.$, $n=3$ mice per group) (Figure 5B). The higher proportion of $\mathrm{CD} 4^{+}$ $\mathrm{CD}^{2}{ }^{+} \mathrm{CD} 95^{+} \mathrm{T}$ cells in DLNs of MECA79-anti-CD3-NP-treated mice suggested a higher rate of activation-induced cellular apoptosis (no treatment vs. free anti-CD3 vs. MECA79-anti-CD3-NP: $38.9 \% \pm$ $5.5 \%$ vs. $35.4 \% \pm 10.2 \%$ vs. $64.7 \% \pm 3.3 \%,{ }^{*} P<0.05, n=3$ mice per group) (Figure 5C). Hearts recovered from the MECA79-anti-CD3NP group contained a significantly lower frequency of $\mathrm{CD}_{4}^{+}$and $\mathrm{CD}^{+}{ }^{+} \mathrm{CD} 69^{+}$(activated) graft-infiltrating $\mathrm{T}$ cells in comparison with the other groups (no treatment vs. free anti-CD3 vs. MECA79-antiCD3-NP: $36.1 \times 10^{4} \pm 7.3 \times 10^{4}$ vs. $21.6 \times 10^{4} \pm 3.8 \times 10^{4}$ vs. $11.5 \times 10^{4}$ $\pm 2.6 \times 10^{4}$ for $\mathrm{CD}^{+} \mathrm{T}$ cells, $33.8 \times 10^{4} \pm 7.3 \times 10^{4} \mathrm{vs} .21 .5 \times 10^{4} \pm 3.8 \times$ $10^{4}$ vs. $13.8 \times 10^{4} \pm 3.9 \times 10^{4}$ for $\mathrm{CD} 4^{+} \mathrm{CD} 69^{+} \mathrm{T}$ cells, ${ }^{*} P<0.05, n=3$ mice per group) (Figure 5D). No difference was noted in the percentages of $\mathrm{CD} 8^{+}$.OTI $\mathrm{T}$ cells among the treatment groups.

Finally, we observed no evidence of either the presence of ectopic HEVs or the infiltration of MECA-NPs in the heart allografts around the time of acute rejection.

A surge of inflammatory cytokines, or cytokine storm, is a major limiting factor in the use of biologic agents, including anti-CD3 $\mathrm{mAb}$ (37). Here, we compared the levels of cytokines in the plasma 2 hours after administration of free anti-CD3 versus MECA79-antiCD3-NP. Our analysis revealed higher levels of inflammatory cytokines, such as IFN- $\gamma$ and IL-2, in the free anti-CD3-treated group in comparison with the MECA79-anti-CD3-NP-treated mice (control vs. free anti-CD3 vs. MECA79-anti-CD3-NP: $3.5 \pm 0.4$ vs. $304.7 \pm$ 54.7 vs. $112.8 \pm 31.5$ for IFN- $\gamma, 3.7 \pm 0.9$ vs. $70.5 \pm 16.5$ vs. $24.5 \pm$ 4.2 for IL-2, ${ }^{*} P<0.05,{ }^{* *} P<0.01,{ }^{* * *} P<0.001,{ }^{* * * *} P<0.0001, n$ $=4$ mice per group, duplicate sample) (Supplemental Figure 4B). No difference was noted in the levels of TNF- $\alpha$ between the free anti-CD3 and MECA79-anti-CD3-NP groups (control vs. free antiCD3 vs. MECA79-anti-CD3-NP: $5.2 \pm 0.9$ vs. $32.6 \pm 4.3$ vs. $43.4 \pm$ 3.1 for TNF- $\alpha, P=\mathrm{NS}, n=4$ mice per group, duplicate sample). This suggests that MECA79-anti-CD3-NP may be better tolerated than free anti-CD3 treatment.

Translating targeted HEV/LN delivery to large animals. Virtually all mammals share similar PNAd molecules expressed by the HEV. To assess the potential of this drug delivery platform to be applied to large mammals, we assessed the efficacy of MECA79-NP in trafficking to the DLN and NDLN in a pig model of vascularized composite allotransplantation. Heterotopic hindlimb transplantation was performed across a full swine leukocyte antigen (SLA) mismatch in MGH miniature swine (ref. 38 and Figure 6A). We injected MECA79-IR800-NPs i.v. into the animal at the time of rejection, and the DLNs and NDLNs were removed 24 hours later. NPs were observed within the vicinity of HEVs in the DLN (Figure 6B). A significantly higher quantity of NPs was observed in the DLN in comparison with the NDLN (NDLN vs. DLN: $0.12 \% \pm 0.03 \%$ vs. $2.28 \% \pm 0.21 \%$, ${ }^{* * * *} P<0.0001, n=5$ LNs per group) (Figure 6C). We next assessed trapping of NP in other organs. While DLN showed the greatest density of MECA79-IR800-NPs, there was also some accumulation in pig liver, and to a lesser extent in the spleen. No MECA79-IR800-NPs were observed in studied sections of lung tissue (Figure 6D). 
A

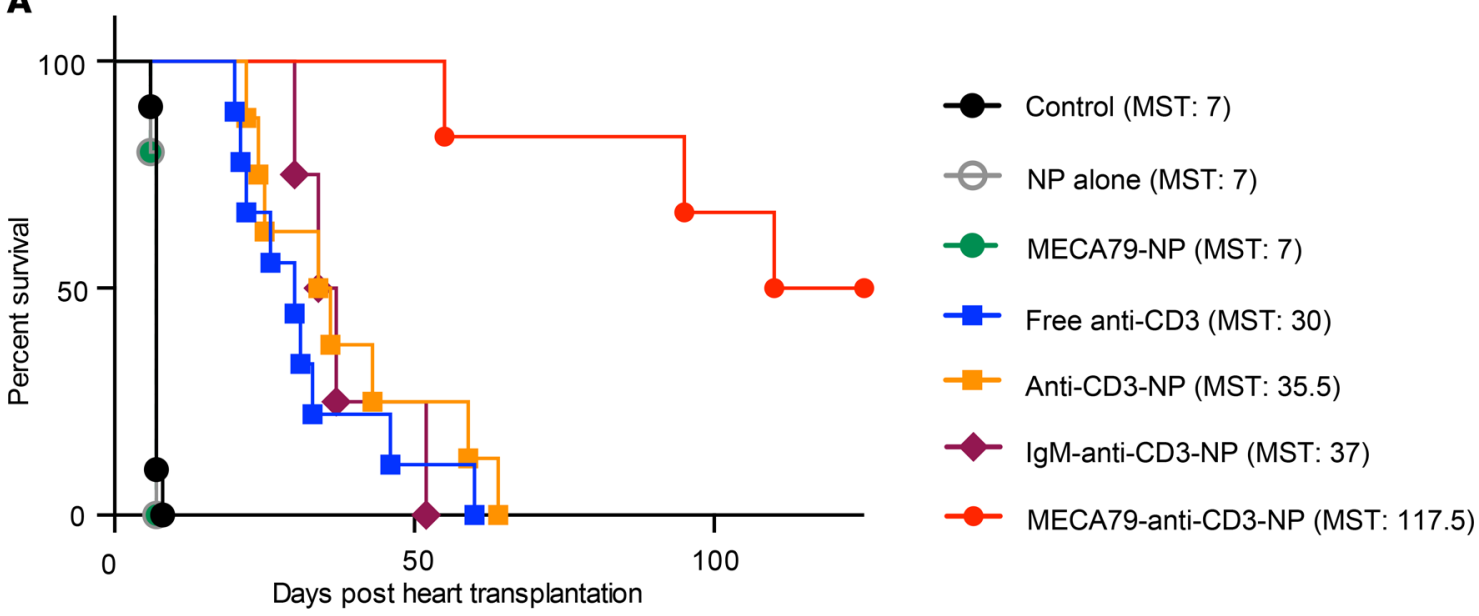

B
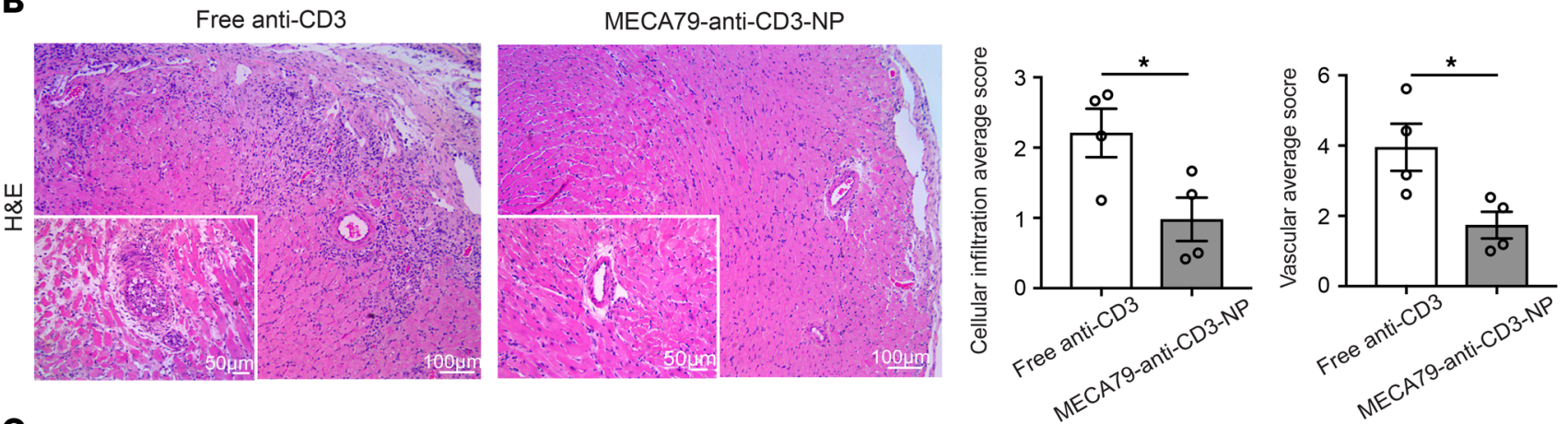

C
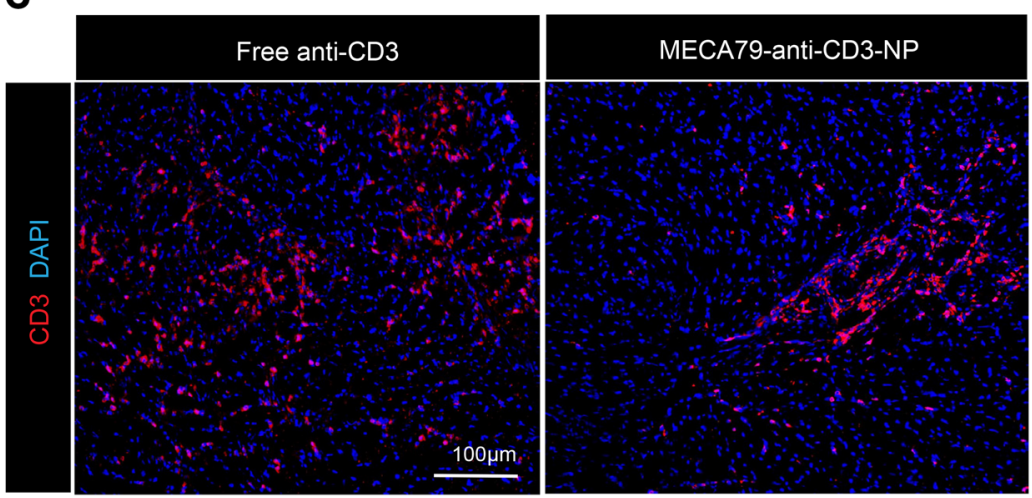

Figure 4. Treatment with MECA79-anti-CD3-NP suppresses acute rejection. $B A L B / c$ hearts were transplanted into C57BL/6 recipients. (A) Survival of heart grafts in recipients of MECA79-anti-CD3-NP treatment (117.5 days) significantly exceeded that in recipients that received no treatment $(7$ days), NP alone (7 days), MECA79-NP ( 7 days), free anti-CD3 (30 days), anti-CD3-NP (35 days), and IgM-anti-CD3-NP (37 days) ( $n=4-9$ mice per group). (B) Representative images of $\mathrm{H} \& \mathrm{E}$-stained heart allografts revealed dense cellular infiltrates in the free anti-CD3 group. The allografts in the MECA79-anti-CD3-NP treatment group showed significantly lower cellular infiltration and lower average vascular scores than those in the free anti-CD3 treatment group
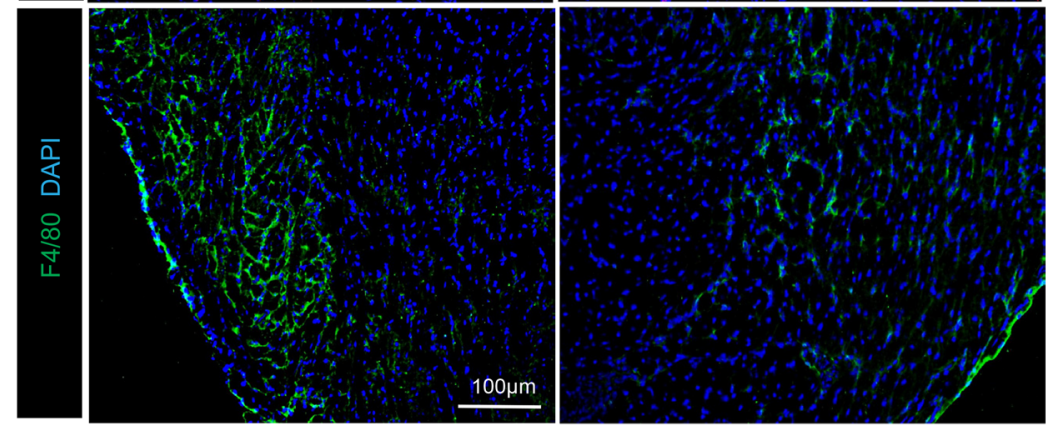
(cellular infiltration score: free anti-CD3 vs. MECA79-antiCD3-NP: $2.209 \pm 0.34$ vs. $0.979 \pm 0.31$, mean \pm SEM, Student's $t$ test, ${ }^{*} P<0.05$; vascular score: $3.954 \pm 0.67$ vs. $1.738 \pm 0.38$, mean \pm SEM, Student's $t$ test, ${ }^{*} P<0.05$; $n=4$ mice per group; bar graphs). (C) Heart grafts treated with MECA79-anti-CD3-NP contained markedly fewer graft-infiltrating $\mathrm{CD}^{+} \mathrm{T}$ cells and $\mathrm{F} 4 / 8 \mathrm{O}^{+}$macrophages in comparison with the free anti-CD3 group, as analyzed using immunofluorescence staining.

\section{Discussion}

In recent years, research studies have focused intently on the concept of LN-targeted drug delivery (11). Targeted delivery of therapeutics allows for the modulation of immune responses and has applications across the entire spectrum of immune-mediated diseases, embodying the potential to either enhance (e.g., conditions such as malignancy/vaccination) or suppress effector activity (e.g., transplantation or autoimmunity) as required (39-46). While the vast majority of these efforts have relied on transmission via absorption through the skin, targeted delivery to HEVs via 
A
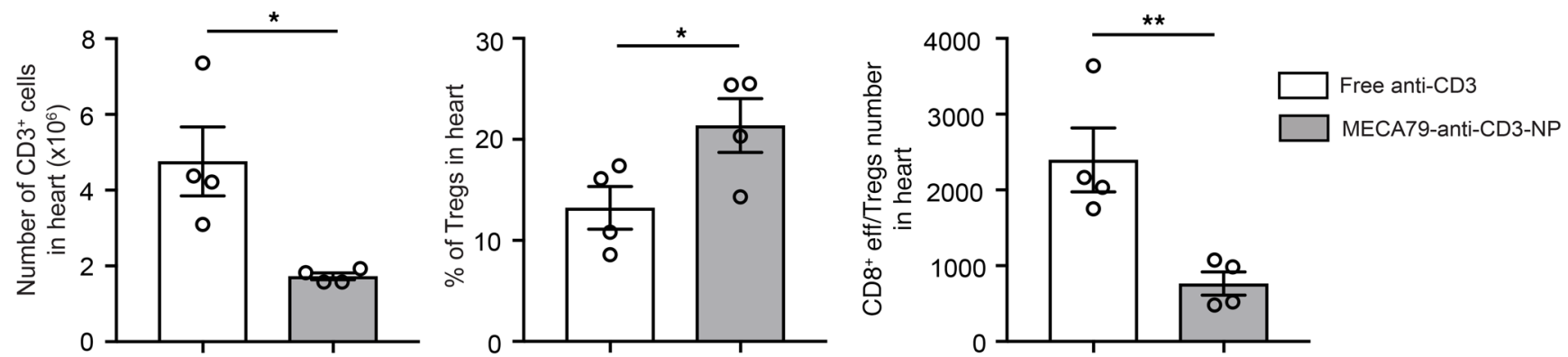

B
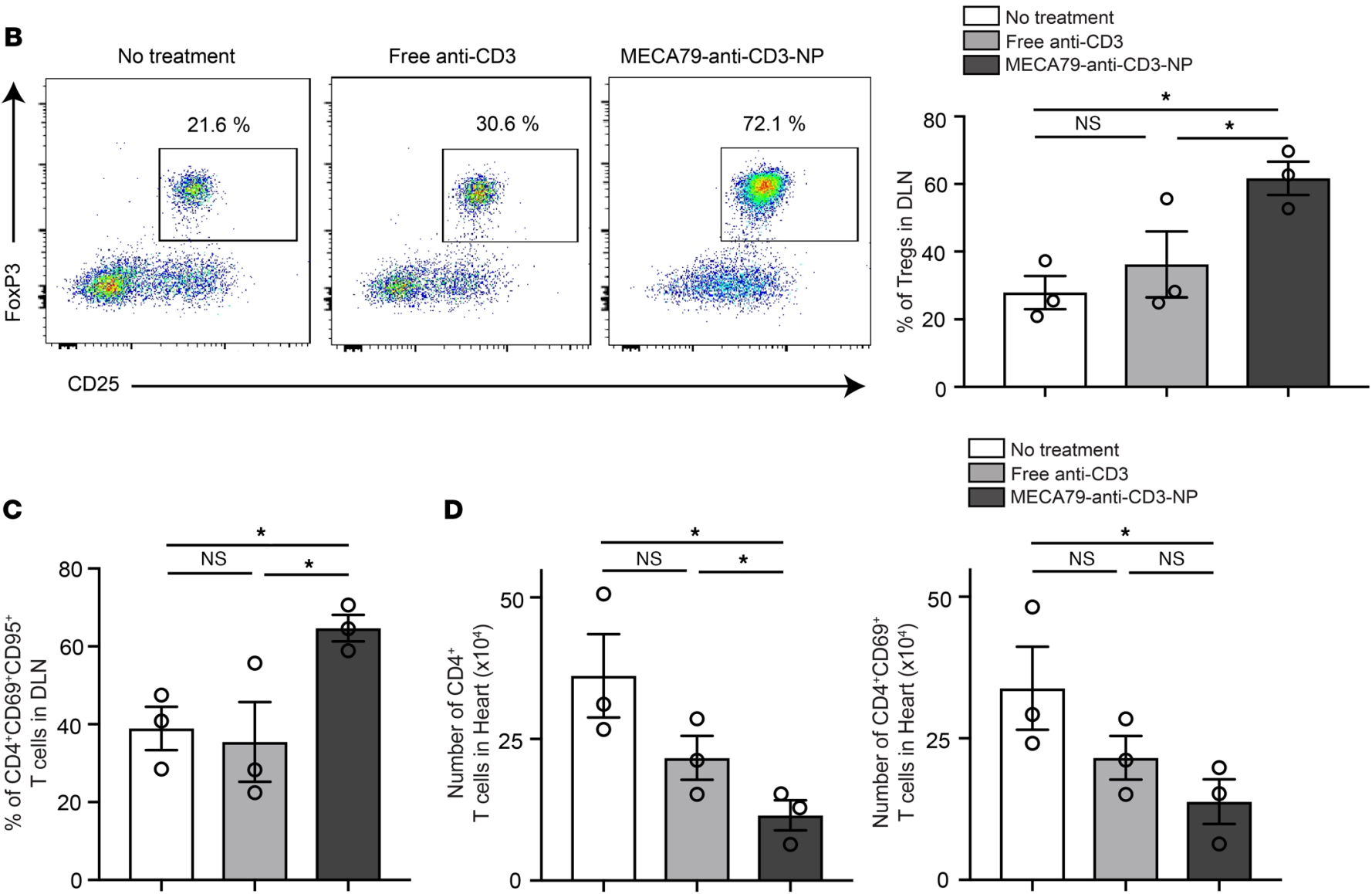

Figure 5. Treatment with MECA79-anti-CD3-NP leads to increased proportion of Tregs in the DLN. (A) BALB/C hearts were transplanted into C57BL/6 recipients and treated with either MECA79-anti-CD3-NP or free anti-CD3. The bar graphs represent the absolute number of CD3+ cells, percentage of Tregs, and ratio of absolute number of CD8 ${ }^{+}$effector T cells to Tregs within heart grafts, harvested at 17 days after transplantation (CD3 ${ }^{+}$cells: free anti-CD3 vs. MECA79-anti-CD3-NP: $4.761 \times 10^{6} \pm 0.9 \times 10^{6}$ vs. $1.728 \times 10^{6} \pm 88,263$, mean \pm SEM, Student's $t$ test, ${ }^{*} P<0.05$; frequency of Tregs: $13.23 \% \pm 2.10 \%$ vs. $21.38 \% \pm 2.65 \%$, mean \pm SEM, Student's $t$ test, ${ }^{*} P<0.05$; ratio of absolute number of CD8 ${ }^{+}$eff/Tregs: $23,97.0 \pm 422.4$ vs. $764.6 \pm 153.8$, mean $\pm S E M$, Student's $t$ test, ${ }^{* *} P<0.01 ; n=4$ mice per group). (B) OTII CD4+ $T$ cells and OTI CD8 ${ }^{+}$T cells were adoptively transferred into Rag ${ }^{-1-}$ mice before OVA heart transplant. DLNs were harvested for analysis at day 5. Flow cytometric analysis shows a significantly higher proportion of Tregs in those treated with MECA79-anti-CD3-NP as compared with the other groups (no treatment vs. free anti-CD3 vs. MECA79-anti-CD3-NP: $27.9 \% \pm 4.8 \%$ vs. $36.2 \% \pm 9.7 \%$ vs. $61.7 \% \pm 4.9 \%$, mean \pm SEM, ANOVA test, ${ }^{*} P<0.05, n=3$ mice per group). Representative flow plots shown. (C) Percentage of CD4 ${ }^{+C D 69+C D 95+}$ T cells in the DLN (no treatment vs. free anti-CD3 vs. MECA79-anti-CD3-NP: $38.9 \% \pm 5.5 \%$ vs. $35.4 \% \pm 10.2 \%$ vs. $64.7 \% \pm 3.3 \%$, mean \pm SEM, ANOVA test, ${ }^{*} P<$ $0.05, n=3$ mice per group). (D) Number of CD4 ${ }^{+}$and $C D 4^{+}$CD69 ${ }^{+}$T cells in the heart allografts (no treatment vs. free anti-CD3 vs. MECA79-anti-CD3-NP: $36.1 \times 10^{4} \pm 7.3 \times 10^{4}$ vs. $21.6 \times 10^{4} \pm 3.8 \times 10^{4}$ vs. $11.5 \times 10^{4} \pm 2.6 \times 10^{4}$ for CD4 $4^{+}$cells, $33.8 \times 10^{4} \pm 7.3 \times 10^{4}$ vs. $21.5 \times 10^{4} \pm 3.8 \times 10^{4}$ vs. $13.8 \times 10^{4} \pm 3.9 \times 10^{4}$ for $\mathrm{CD}^{+}{ }^{+} \mathrm{CD}_{69}{ }^{+} \mathrm{T}$ cells, mean $\pm \mathrm{SEM}$, ANOVA test, ${ }^{*} P<0.05, n=3$ mice per group).

systemic administration provides an unprecedented opportunity to significantly broaden the clinical applications of targeted LN therapies, particularly for conditions in which the LN is not easily accessible from the skin $(39,45,47,48)$. The unique expression of
PNAd by HEVs marks them as exquisite targets for drug delivery, especially with respect to the limitation of off-target toxicity.

We have previously used MPs for delivery of tacrolimus to the LNs. For our current studies, we have engineered a formula- 
A
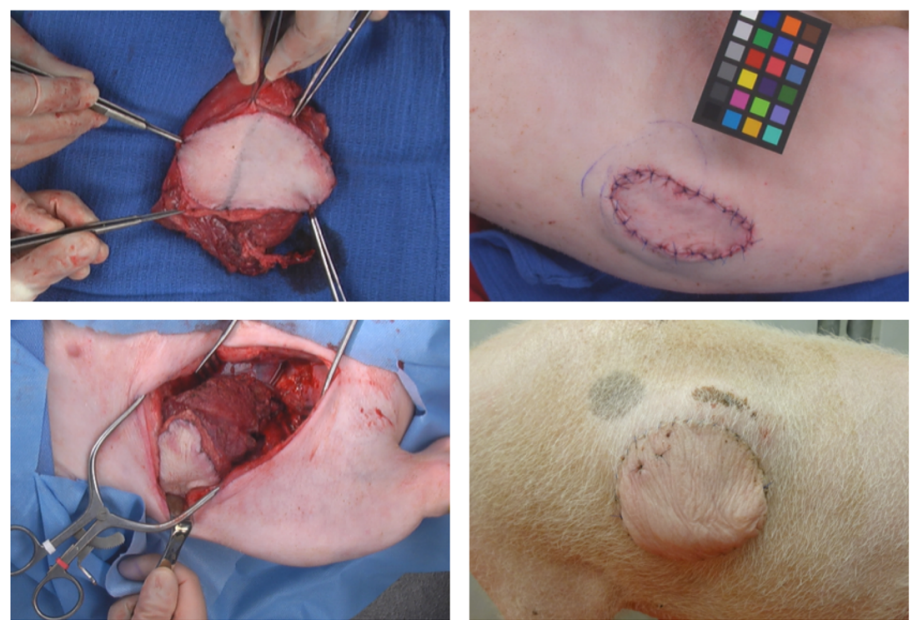

\section{B}

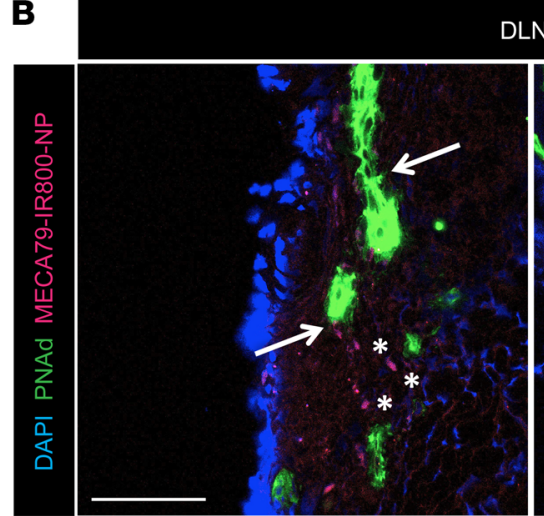

LN

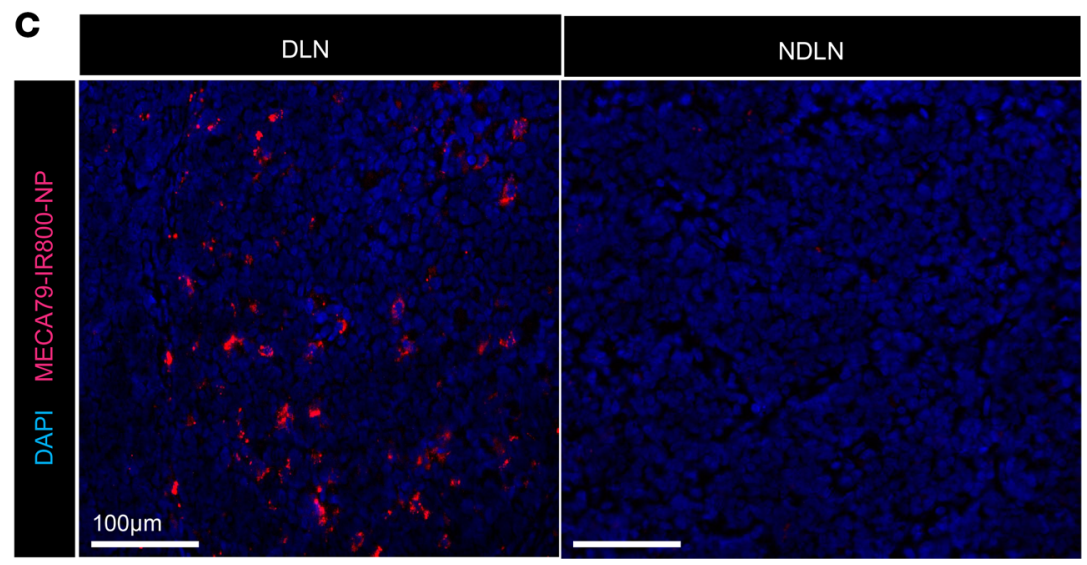

Figure 6. MECA79-NP traffic to the DLN of a pig with a vascu-

larized allogeneic limb transplant. (A) Representative figures of vascularized composite allotransplant (VCA) in swine leukocyte antigen-defined (SLA-defined) MGH miniature swine. Top left: Dissection of VCA containing skin, muscle, and bone from the donor hind limb. Bottom left: Inset of the VCA into the groin and lateral abdominal wall of the recipient animal. Top right: Final skin inset in the lateral abdominal wall. Bottom right: Long-term follow-up by visual monitoring of the donor skin component in the lateral abdominal wall of the recipient. (B-D) MECA79IR800-NP was injected i.v. 147 days after VCA. DLN, NDLN, liver, spleen, and lung were harvested at 24 hours after injection. (B) Immunofluorescence staining of DLN. Arrows point to the MECA79-IR800-NPs in the proximity of the HEVs in the DLN.

Scale bars: $100 \mu \mathrm{m}$. (C) Immunofluorescence staining of DLN and NDLN. The bar graph shows percentage area per each image positive for MECA79-IR800-NPs, quantified using Imagel software (NIH). A significantly higher quantity of NPs was observed in the DLN as compared with the NDLN (NDLN vs. DLN: $0.12 \% \pm$ $0.03 \%$ vs. $2.28 \% \pm 0.21 \%$, mean \pm SEM, Student's $t$ test, ${ }^{* * *} P$ $<0.001, n=3$ images per section, total 5 sections per $L N$ ). (D) Immunofluorescence staining of liver, spleen, and lung displays the preferential accumulation of MECA79-IR800-NPs in the liver and spleen, as demonstrated by asterisks.
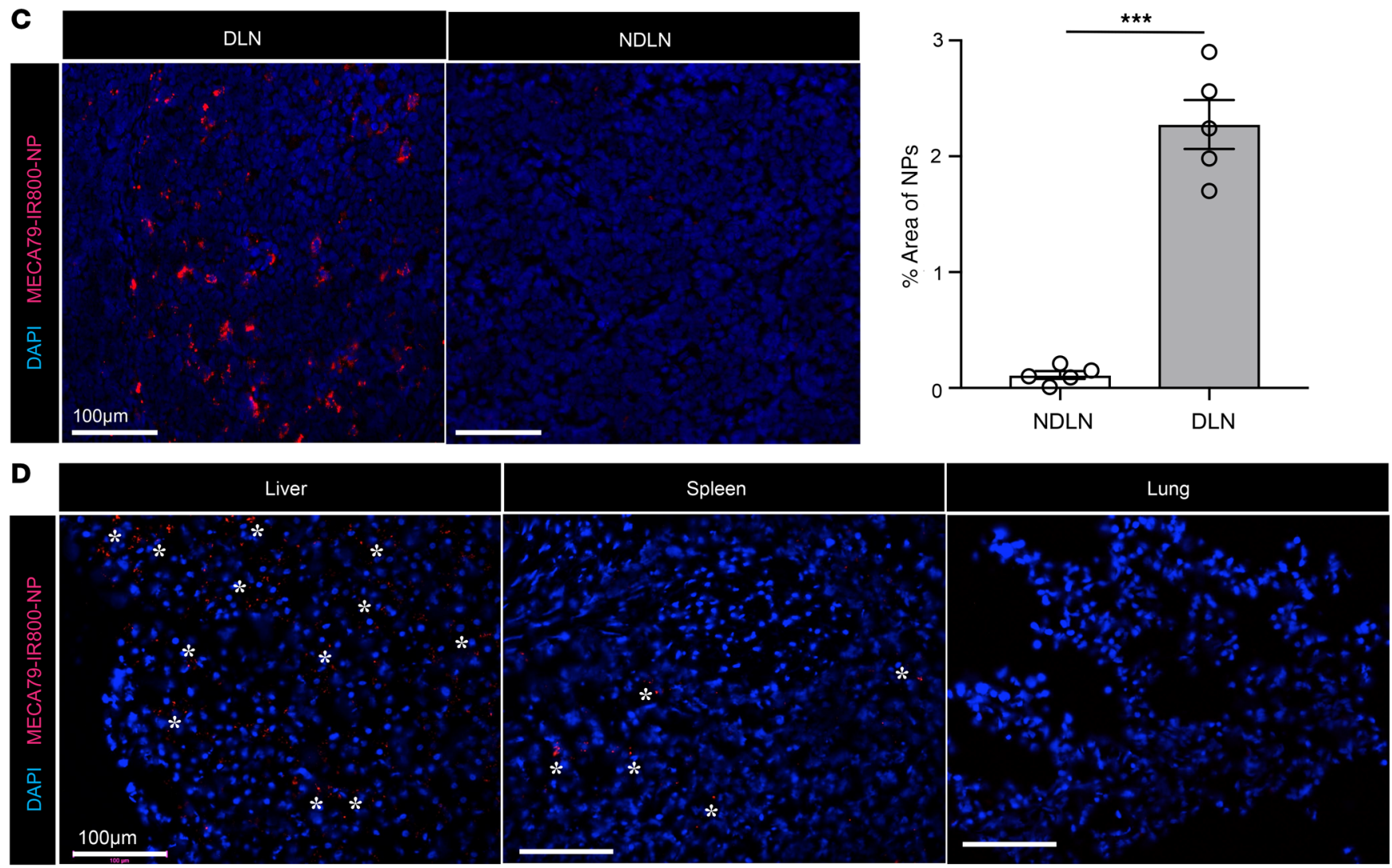
tion of NPs with particular physicochemical properties that serve to increase circulation time. These NPs are slightly negatively charged and uniformly spherical with an average size of approximately $100 \mathrm{~nm}$, which leads to a reduced rate of clearance by the mononuclear phagocyte system. In contrast, positively charged NPs undergo more rapid uptake by macrophages in the lung, spleen, and liver (49), and nonspherical NPs are sequestered more readily by the mononuclear phagocyte system. In terms of size, while the lungs entrap large particles such as MPs, the kidneys clear very small $(<10 \mathrm{~nm})$ particles readily. Therefore, NPs with characteristics such as ours have a prolonged circulation time. Furthermore, we previously used protein $\mathrm{G}$ to conjugate MECA79 to our MP, which is a noncovalent molecule and has reversible binding. However, protein $\mathrm{G}$ may bind nonspecifically and induce an immune reaction in vivo (50). The new antibody conjugation method used in this study uses covalent bonding, confers site specificity, and provides control over the orientation of the antibody conjugated to the NP (11).

Our conjugation technique relies on cleaving the thiols of MECA79 mAb (51). In contrast to IgG, which has disulfide bonds at the hinge region, most of the disulfide bonds of the intrachain region of $\operatorname{IgM}$ are more distal, a position that is responsible for its pentamer shape and yields more intact antibodies following reduction (52-57). The finding that conjugation strategies result in heterogeneous groups of antibody products is well recognized (24, $25,58)$. In the setting of heterogeneity, the nonrelevant products may compete with the monomer at the time they are incorporated on the surface of the NP. They could also impact the orientation of monomers or compete with the binding sites at the level of the HEV. Future studies using cryo-electron microscopy could more clearly delineate the physical orientation of antibody products on the surface of the NP (59). However, using our conjugation technique, we found efficient trafficking of MECA79-NPs to LNs, indicating that by choosing monomers, our method may result in higher loading and more controlled orientation of MECA79 mAb on the surface of the NP. Other strategies target the lysine residue of the antibody; however, the abundance of lysine residues on the surface of many proteins can result in random conjugation and a heterogeneous product profile (60). Other site-specific conjugation techniques require multistep purification, which complicates the preparation of the drug (61). The simplicity of our conjugation methodology enhances its clinical translatability.

Another novel aspect of our current delivery platform centers on the importance of anti-CD3 as an induction therapy. This platform is especially suited to increase the efficacy of transplantation induction therapy and to reduce the toxicity of immunosuppressive drugs. In contrast to tacrolimus, which requires long-term therapy after transplantation, the application of nanomedicine to short-course induction therapy is clinically more feasible. Using highly potent and selective agents in the initial phase of transplantation has reduced the incidence of acute rejection $(26,27)$ and emerged as the standard of care for transplant patients $(28,29)$. However, the use of induction regimens is associated with an increased risk of serious infection, malignancy (62-64), diabetes, and dyslipidemia (65). Our strategy may greatly improve the safety profile of induction immunosuppression by enabling significantly lower systemic dosing, while maintaining clinical efficacy. Notably, similar induction therapies are used for a wide variety of life-threatening immunemediated diseases, in which the LN occupies an important role as a primary site of immune activation, indicating a broad range of potential applications for this treatment approach.

Our trafficking studies have demonstrated the targeted accumulation of MECA79-NPs in the DLN, which contains expanded $\mathrm{HEVs}$ and receives increased blood flow following transplantation. We observed sequential migration of NPs into the LN interstitium over time. DCs and T cells within the DLN internalized the vast majority of NPs. While the total amount of anti-CD3 present in the DLN was increased in mice treated with MECA79-anti-CD3$\mathrm{NP}$, a much higher percentage of $\mathrm{T}$ cells bound with fluorescently labeled anti-CD3 was also found, indicating successful targeting within the LN. As DCs and T cells reside within the vicinity of the HEV, these cells may internalize the NPs along their pathway of migration into the parenchyma of the LN and subsequently release anti-CD3. Anti-CD3 released from NPs taken up by DCs within the LN may also bind to the dense population of locally resident $\mathrm{T}$ cells. Therefore, we believe this delivery platform increases the accessibility of anti-CD3 to T cells.

We noted a robust augmentation of Tregs within the heart allografts of mice treated with MECA79-anti-CD3-NP, but were unable to demonstrate augmentation of Tregs within the DLN in a polyclonal transplant model. This negative finding could be due to the presence of transient immune regulation a priori during the formation of Tregs, which migrate later to the allografts. The kinetics of the effect of anti-CD3 on immune cells has been reported to be heavily dependent on the timing of antiCD3 dosing, so the immunoregulatory effects of anti-CD3 could shift with time (66). Nonetheless, Wood and colleagues noted that the augmentation of intragraft Tregs accounted for much of the immunosuppressive effect they attributed to anti-CD3 (66). The promotion of Tregs by anti-CD3 mAbs through the actions of IL-2, TGF- $\beta$, and IL-7 has been examined extensively and described in detail (32-34). Resident Tregs have been reported to survey the environment of the tissue (66), and indeed in our model we observed a marked increase in apoptosis among activated $\mathrm{T}$ cells within the heart, likely reflecting the action of Tregs in the allograft. Depletion of Tregs with anti-CD25 in our study abrogated the prolongation of heart graft survival. Similarly, Kendal et al. have demonstrated that when the intragraft FoxP3 ${ }^{+}$ Tregs were depleted from allografts, they were rejected without the need for effector T cells (67). In our model, MECA79-antiCD3-NP likely had a transient intra-LN effect leading to induction of Tregs, which then migrated to the allograft. The small amount of anti-CD3 used in these models could explain our findings that the T cells were not depleted significantly, and the T cell repertoire did not change between the treatment groups.

The survival of the heart allografts in LT $\alpha-\mathrm{KO}$ recipients demonstrated the importance of the $\mathrm{LN}$ as a site of immune regulation following treatment with MECA79-anti-CD3-NP. Our kinetic data showed comparable trafficking of MECA79-conjugated as well as nonconjugated NPs to the spleen. However, splenectomy did not abrogate the therapeutic effects of MECA79-anti-CD3-NP. These findings suggest that the spleen does not play a predominant role in the prolongation of heart allograft survival in these mice. 
One important complication of anti-CD3 is the release of cytokines that lead to the generation of Fc-modified anti-CD3 (68). Examination of the early release of cytokines revealed evidence of a higher surge of inflammatory cytokines, such as IFN- $\gamma$ and IL-2, in the blood of the mice treated with free anti-CD3, as compared with MECA79-anti-CD3-NP (37, 69-71). No difference was observed with respect to the level of TNF- $\alpha$, which could be attributed to the typical timing surrounding the release of TNF- $\alpha$ release (72). This finding suggests that along with increased efficacy of anti-CD3 using this delivery platform, the slow release from MECA79-anti-CD3-NP may also be better tolerated than standard anti-CD3 dosing.

An important aspect of these studies is that $\mathrm{HEV}$ vasculature expresses PNAd constitutively in virtually all LNs (73). Therefore, targeting this molecule may deliver immunomodulatory molecules to all LNs, which could result potentially in generalized immunosuppression. In our model, the density of HEVs increased in the DLN following transplantation, which resulted in local accumulation of MECA79-anti-CD3-NPs at the site of immune activation. Furthermore, while this possibility requires full investigation, we believe that the significant reduction in the overall dosing of immunosuppressive agents reduces the likelihood of systemic toxicity related to immunosuppression, including the risk of opportunistic infections.

Finally, the power in this platform lies in its specificity of $\mathrm{LN}$ targeting and the capacity to load NPs with varied immunosuppressive agents, opening up the possibility of targeting a wide range of diseases. The potential applications of an HEV-targeted drug delivery platform include immunotherapy for diseases such as autoimmune diabetes - in which the pancreatic lymph node is the primary site of autoreactivity - and malignances such as primary lymphomas, as well as the creation of an HEV-targeted paradigm of molecular imaging.

\section{Methods}

Self-assembly, single-step synthesis of IR800-NPs. The PLGA copolymers were purchased from PolySciTech, Akina. For each NP synthesis, methoxy poly(ethylene glycol)- $b$-poly(D,L-lactic-co-glycolic) acid copolymer (mPEG-PLGA; MW 5,000:30,000 Da, 50:50 LA/GA [wt/ wt]) and poly(lactide-co-glycolide)- $b$-poly(ethylene glycol)-maleimide (maleimide-PEG-PLGA; MW 30,000:5,000 Da, 50:50 LA/GA) were used as the core polymers. NPs were engineered using self-assembly single-step nanoprecipitation. PEG-PLGA and maleimide-PEG-PLGA were dissolved in acetone. The IRDye 800CW (LI-COR; dissolved in methanol, $2.5 \mathrm{mg} / \mathrm{ml}$ ) was added to the polymer mixture, vortexed, and then added dropwise to $20 \mathrm{ml}$ of distilled water. The NP suspension was stirred for 2 hours and was concentrated by centrifugation using Amicon Ultra-15 centrifugal filter units (MW cutoff [MWCO] 100 kDa; SigmaAldrich) at $1,800 \mathrm{~g}$ in intervals of 5 minutes. The resulting NPs were washed with deionized water and resuspended in $1 \mathrm{ml}$ of distilled water.

Synthesis of anti-CD3-NPs. Anti-CD3-NPs were synthesized using a double-emulsion/solvent evaporation method. PEG-PLGA and maleimide-PEG-PLGA were dissolved in ethyl acetate. The anti-CD3 $\mathrm{mAb}$ (rat monoclonal anti-mouse CD3, clone 17A2, catalog 14-003282, Thermo Fisher Scientific) was diluted in PBS and added to the polymer mixture, vortexed on high speed for 1 minute, and sonicated for 1 minute in a bath sonicator. The first emulsion was then added dropwise to $3 \mathrm{ml}$ of distilled water while being vortexed on high speed, followed by 1 minute of sonication in bath sonicator. The resulting suspension of NPs was added dropwise to $20 \mathrm{ml}$ of distilled water and stirred for 2 hours to evaporate the organic phase. Anti-CD3-NPs were concentrated by centrifugation using Amicon Ultra-15 centrifugal filter units (MWCO $100 \mathrm{kDa}$; Sigma-Aldrich) at 3,750 rpm in intervals of 5 minutes. The resulting NPs were washed with deionized water and resuspended in $1 \mathrm{ml}$ of distilled water.

Conjugation of MECA79. The MECA79 mAb (NB100-77673, NOVUS Biologicals) was conjugated to the functional surface of NPs using thiol-maleimide chemistry. Thirty microliters of MECA79 $\mathrm{mAb}(1.0 \mathrm{mg} / \mathrm{ml})$ was pretreated with $30 \mu \mathrm{lTCEP}(0.5 \mathrm{M}, 15$ minutes, room temperature) to cleave thiol groups and was mixed immediately with the suspension of NPs. Maleimide groups on NPs bind covalently to the cleaved thiol of the MECA79 antibody. The MECA79-NPs were stored at $4^{\circ} \mathrm{C}$ before use.

Characterization of NPs. The size and surface charge of NPs were measured using dynamic light scattering. The NPs were diluted 100fold for characterization tests. The morphology of NPs was observed using transmission electron microscopy. Freshly prepared NPs were deposited on 200-mesh Formvar/carbon-coated copper grids, washed with distilled water, and negatively stained with $0.75 \%$ uranyl formate stain. The samples were imaged immediately with a Tecnai G2 Spirit BioTWIN electron microscope equipped with an AMT 2k CCD camera and low-dose software $(80 \mathrm{kV}$, direct magnification $\times 98,000)$. A calibration curve of various concentrations of the anti-CD3 mAb was prepared using an ultraviolet-visible (UV-VIS) spectrophotometer to quantify the amount of antibody loaded in the NPs. The absorbance of the samples measured at $275 \mathrm{~nm}$ was compared with the calibration curve, in order to determine the loading efficiency of anti-CD3 mAb.

Release kinetics of anti-CD3. To quantify the release profile of anti-CD3 mAb from the NPs, anti-CD3-NPs were incubated at $37^{\circ} \mathrm{C}$ in triplicate. At defined durations of incubation ( 2 hours, 6 hours, and 1, 2, 3, 4, 7, and 14 days), the NPs were removed, transferred to Amicon Ultra-15 centrifugal filter units (MWCO 10 kDa; SigmaAldrich), and centrifuged at 3,750 rpm for 5 minutes. The filtrate (10 $\mu \mathrm{l})$ was analyzed with a UV-VIS spectrophotometer, and absorbance was measured at $275 \mathrm{~nm}$ to determine the amount of released antibody at each time point.

Optimization of MECA79 conjugation. To study the effect of the reducing agent on the structural integrity and activity of MECA79 antibody, we varied the ratio of TCEP to MECA79 over a wide range (10:2, 30:2, 30:6, and 30:30) and studied the trafficking of MECA79IR800-NPs to the LNs in vivo. A nonreducing gel was used to analyze the MW of reduced MECA79 mAb. MECA79 mAb was incubated with various concentrations of TCEP for 15 minutes and was loaded immediately into the gel before running. Nonreduced MECA79 mAb and isotype $\operatorname{IgM}$ were used as controls.

Mice. C57BL/6J (H-2 $\left.{ }^{\mathrm{b}}\right), \mathrm{BALB} / \mathrm{c}\left(\mathrm{H}-2^{\mathrm{d}}\right), \mathrm{C} 57 \mathrm{BL} / 6.129 \mathrm{~S} 7-\mathrm{Rag}^{\text {tmIMom }}$ ( $\left.\mathrm{Rag}^{-/}\right), \mathrm{C} 57 \mathrm{BL} / 6-\mathrm{Tg}(\mathrm{CAG}-\mathrm{OVA}) 916$ Jen (OVA), C57BL/6-Tg(TcraTcrb) 1100Mjb (OTI), C57BL/6-Tg(TcraTcrb)425Cbn (OTII), and B6.129S2$\mathrm{Lta}^{\mathrm{tm} 1 \mathrm{Dch}} / \mathrm{J}(\mathrm{LT} \alpha-\mathrm{KO})$ mice were obtained from The Jackson Laboratory. Male or female mice were used at 8-10 weeks of age and were housed in sterilized, ventilated cages in a specific pathogen-free animal facility under a standard 12-hour light/12-hour dark cycle. Each experiment was performed using 3-9 mice per group. All animal experiments and methods were performed in accordance with the relevant guidelines and regulations 
approved by the Institutional Animal Care and Use Committee of Brigham and Women's Hospital, Harvard Medical School.

Mouse heart transplantation. Vascularized intra-abdominal heterotopic transplantation of heart allografts was performed using microsurgical techniques, as described previously (74). The heart was harvested following the ligation/dissection of superior vena cava and inferior vena cava, and dissection of ascending aorta and pulmonary artery from the donor mouse. The harvested donor heart was stored at $4^{\circ} \mathrm{C}$ and immersed in UW (University of Wisconsin) solution until transplantation (within 30 minutes). Following abdominal incision of the recipient mouse, the abdominal aorta and inferior vena cava were clamped. The ascending aorta and pulmonary artery of the donor heart were sutured to the abdominal aorta and inferior vena cava of the recipient mouse, respectively, using a 10-0 suture. Contractions of transplanted heart were observed upon removal of the cross-clamp, and the abdominal incision was closed in 2 layers with a 6-0 silk running suture. For splenectomy, the splenic artery and vein were tied with a 6-0 silk suture and removed at the time of heart transplantation. The survival of cardiac allografts was assessed by daily palpation. Rejection was defined as complete cessation of cardiac contractility, as determined by direct visualization.

Skin transplantation. BALB/c donor trunk skin graft $\left(1 \mathrm{~cm}^{2}\right)$ was transplanted onto the left upper back of a C57BL/6 recipient mouse. Skin allografts were sutured with 6-0 silk and secured with a bandage for 7 days.

In vivo biodistribution studies of NP. C57BL/6 mice were used for biodistribution studies. The mice were prepared for imaging by shaving of the abdominal area and underarms. The samples were administered i.v. via retro-orbital injection. Trafficking of fluorescent MECA79-IR800-NPs was studied using a UVP iBox Explorer Imaging Microscope equipped with a 750-to-780-nm excitation filter and an 800-nm long-pass emission filter. The mice were anesthetized before imaging and placed in the dark box in a supine position. LNs and organs, such as heart, lung, liver, and spleen, were collected and analyzed for the evaluation of the biodistribution of the NP.

Color Doppler echocardiography of LNs. Naive and C57BL/6J mice at 8 days after allogeneic skin transplant were anesthetized using $1 \%-3 \%$ isoflurane and $100 \%$ oxygen, and placed on a heating pad. Hair was removed before imaging. The mouse was monitored by echocardiogram and rectal thermometer. Color Doppler echo (Vevo 2100, Fujifilm) was used to observe the axillary LN, and a video recording was taken. The volumetric flow rate was analyzed in each frame of the recording, and the mean flow volume was obtained.

Cannulation of thoracic duct and collection of lymph fluid. Seven hundred microliters of olive oil was administered to mice orally 30 minutes before the operation. The left abdomen was opened through a left-sided incision, and a self-retractor was placed. The lymph duct was dissected carefully from the abdominal aorta and the surrounding tissue, as described previously (75). A silastic silicone tube was tunneled subcutaneously and exteriorized through the back of the neck by an 18-gauge needle. The lymph duct was transversely opened by microscissors, and a silicone tube was inserted into the thoracic duct caudally and fixed by wound glue. The abdominal wall was closed in 2 layers with a 6-0 silk running suture. The lymph fluid was collected in an Eppendorf tube via the silicone tube for up to 5 hours. The lymph fluid was smeared on the glass slide and fixed with formalin for observation by light microscopy.
Extraction of lymphocytes from transplanted heart grafts. Heart grafts were procured and flushed with PBS to remove any remaining clot. They were then minced in RPMI 1640 medium containing $0.1 \%$ collagenase and incubated for 1 hour at $37^{\circ} \mathrm{C}$ with $5 \% \mathrm{CO}_{2}$, followed by addition of $0.1 \mathrm{M}$ EDTA in PBS. After 5 additional minutes of incubation at $37^{\circ} \mathrm{C}, 5 \mathrm{mM}$ EDTA in PBS with $1 \%$ of FBS buffer was added. Cells were then filtered through a $70-\mu \mathrm{m}$ cell strainer, counted, and stained for flow cytometry.

In vivo depletion of Tregs. To assess the role of Tregs in heart transplant survival, Tregs were depleted with anti-CD25 antibody (200 $\mu$ i.v. per mouse; LEAF Purified anti-mouse CD25, 102031, clone PC61, BioLegend) at 25 days after heart transplant (BALB/c heart into C57BL/6 recipient, treated with MECA79-anti-CD3-NP).

Staining for $T$ cell repertoire. PE anti-mouse TCR V $\alpha 2$ antibody (127807, BioLegend), APC/Cy7 anti-mouse TCR $\beta$ chain antibody (129219, BioLegend), and PerCP/Cy5.5 anti-mouse TCR $\gamma / \delta$ antibody (118117, BioLegend) were used to analyze T cell repertoire.

Histological and immunohistochemical assessments. Five-micrometerthick formalin-fixed, paraffin-embedded heart sections were stained with standard H\&E stain. Histological evaluation was done using a score modified from the International Society for Heart and Lung Transplantation $(35,36)$. Lymphocyte cellular infiltration was graded blindly from 0 to 5 in 6 random fields of each H\&E section ( 5 sections per heart, 4 mice per group). The grades were defined as follows: grade 0 , no cellular infiltration; grade 1 , less than $25 \%$ cellular infiltration; grade 2, 25\%-50\% cellular infiltration; grade 3, 50\%-75\% cellular infiltration; grade 4 , greater than $75 \%$ cellular infiltration; and grade 5 , myocyte hemorrhage or scar. The vascular score is determined by a combination of the vascular occlusion score and perivascular cellular infiltration. Vascular occlusion is scored from grade 0 to 4 for every vessel ( 5 sections per heart, 4 mice per group). The grades are defined as follows: grade 0 , no occlusion; grade 1 , less than $50 \%$ occlusion; grade $2,50 \%-75 \%$ occlusion; grade 3, 75\%-95\% occlusion; and grade 4, more than $95 \%$ occlusion. The perivascular cellular infiltration is scored as follows: grade 0 , no cellular infiltration; grade 1 , less than $25 \%$ cellular infiltration; grade 2, 25\%-50\% cellular infiltration; grade 3, 50\%-75\% cellular infiltration; and grade 4 , greater than $75 \%$ cellular infiltration. Then, the vascular occlusion score and perivascular cellular infiltration score were totaled as the vascular score.

Immunofluorescence staining of tissues. Frozen OCT blocks of tissues were cut using a cryotome into 8 - $\mu \mathrm{m}$-thick sections and stained using MECA79 (NOVUS Laboratories), CD31 (390, eBioscience), CD3 (SP7, Abcam; or 17A2, eBioscience), CD11b (M1/70, BioLegend), CD11c (N418, eBioscience), B220 (RA3-6B2, eBioscience), and F4/80 (BM8, eBioscience) antibodies. The stained tissue sections were imaged using a fluorescent confocal microscope and an EVOS FL Auto 2 Imaging System (Thermo Fisher Scientific). DAPI (VECTASHIELD, Vector Laboratories) was used to stain the cell nuclei.

Flow cytometry. Flow cytometric analysis of graft-infiltrating cells, DLN, and spleen was performed, and each leukocyte population was quantified. The spleen was placed onto a $70-\mu \mathrm{m}$ cell strainer (BD Falcon) attached to a 50-ml conical tube. The spleen was mashed in sterile Dulbecco's Phosphate-Buffered Saline (DPBS) through the strainer using the plunger end of a syringe. The single-cell suspension was centrifuged at $340 \mathrm{~g}$ for 5 minutes. The pellet was resuspended in $1 \mathrm{ml} \mathrm{ACK} \mathrm{lysis}$ buffer (Lonza) and incubated for 2 minutes. The lysing reaction was stopped by addition of $30 \mathrm{ml}$ DPBS, and the cell suspension was cen- 
trifuged at 1,600 rpm for 5 minutes. The pellet was resuspended in complete RPMI 1640 at $1 \times 10^{7}$ cells $/ \mathrm{ml}$. The same protocol was used to prepare a single-cell suspension from LNs, omitting the lysing step. All antibodies were purchased from BD (Becton Dickinson). Cells were run on a FACSCanto II (BD Biosciences) instrument. Data were analyzed using FlowJo software.

Western blot assay. Whole LN cell lysates were measured using the Bradford assay. Equal amounts of protein were separated by SDSPAGE and transferred to a PVDF membrane. The membranes were immunoblotted with the following specific antibodies: anti-rat IgGHRP (Sigma-Aldrich) and anti- $\beta$-actin (Sigma-Aldrich), using standard protocols. The blots were developed with West Dura chemiluminescent substrates using a Bio-Rad ChemiDoc imaging system.

$T$ cell assay. Splenocytes were isolated from the spleen of a C57BL/6 mouse and labeled with a CellTrace Violet kit (catalog C34557, Thermo Fisher Scientific). Next, cells were seeded at 1 million cells per well in a round-bottom 96-well plate. Free anti-CD3 $(10 \mu \mathrm{g})$ and anti-CD3 NPs (dose equivalent to $10 \mu \mathrm{g}$ anti-CD3) were added to the cells. Splenocytes that were not treated with any reagent were considered as the negative control. The plate was placed in a humidified $37^{\circ} \mathrm{C}, 5 \% \mathrm{CO}_{2}$ incubator for 3 days. Cells were analyzed using a FACSCanto-II flow cytometer (BD Biosciences) and FlowJo software version 9.3.2 (Tree Star).

Incubation of DCs and T cells with ${ }^{*}$ anti-CD3. CD11 $\mathrm{c}^{+} \mathrm{DCs}$ and $\mathrm{CD}^{+} \mathrm{T}$ cells were isolated from spleens of C57BL/6 mice using the EasySep Mouse CD11c Positive Selection Kit II and the EasySep Mouse T Cell Isolation Kit (STEMCELL Technologies), respectively. CD11 $c^{+}$DCs $(150,000$ cells per well) were cultured in complete RPMI 1640 medium (Lonza), supplemented with 10\% FBS (Gemini Bio-Products), $1 \%$ penicillin/streptomycin (Corning), and $1 \%$ L-glutamine (Corning), and treated with Alexa Fluor 488 anti-mouse CD3 ( ${ }^{*}$ anti-CD3) or Alexa Fluor 488 anti-mouse CD3-NPs ( ${ }^{*}$ antiCD3-NPs). After 1 hour of incubation, CD11 $\mathrm{c}^{+}$DCs were washed twice with DPBS, and $\mathrm{CD}^{+} \mathrm{T}$ cells $(450,000$ cells per well) were added to these cells. The cells were incubated together for 6 hours at $37^{\circ} \mathrm{C}$ in a humidified atmosphere containing $5 \% \mathrm{CO}_{2}$. Then, the $\mathrm{T}$ cells were examined by flow cytometric analysis.

Swine heterotopic limb transplantation. Swine heterotopic hindlimb transplant procedure was performed as reported previously (38). Briefly, the donor hind-limb allograft was placed into a pocket in the abdominal wall of the recipient animal, and end-to-end femoral artery and vein anastomosis was performed, rendering the ipsilateral exter- nal iliac LNs as the DLNs of the allograft. At the time of clinically visible rejection of the skin component of the allograft, we intravenously injected MECA79-IR800-NPs, and DLNs (ipsilateral external iliac LNs) and non-DLNs (contralateral external iliac LNs) were removed 24 hours later to assess localization of NPs.

Statistics. Kaplan-Meier survival graphs were constructed, and a log-rank comparison of the groups was used to calculate $P$ values. Two-way ANOVA and 1-way ANOVA with Tukey's or Dunnett's multiple-comparisons post-test analysis and unpaired 2-tailed $t$ test were used for comparison of experimental groups. Differences were considered to be significant for ${ }^{*} P<0.05,{ }^{* *} P<0.01$, and ${ }^{* *} P<0.001$. Prism software was used for data analysis and to prepare graphs (GraphPad). Data represent mean \pm SEM.

Study approval. All experiments were performed according to the animal experimental guidelines upon the approval of the Institutional Animal Care and Use Committee at Brigham and Women's Hospital (Boston, Massachusetts, USA).

\section{Author contributions}

BB designed and fabricated NPs, designed and performed experiments, analyzed data, and wrote the main text of the manuscript. MU designed and performed experiments and microsurgery, analyzed data, and wrote parts of the Methods and Results sections. LJ, FO, NB, TI, ZS, GJF, DA, IV, and OAY performed experiments. GB, UHVA, KU, QX, YH, JA, VK, JSB, and MMM helped with study design and critically revised the manuscript. RA designed the study, interpreted and analyzed data, and critically revised and finalized the manuscript.

\section{Acknowledgments}

This work is in part supported by NIH grant T32-EB016652 (to $\mathrm{BB})$, National Institute of Allergy and Infectious Diseases grants R01-AI126596 and R01-HL141815 (to RA), aa Brigham Research Institute Stepping Strong Innovator Award (to RA), the BWH Health \& Technology Innovation Fund (to RA), and National Priorities Research Program (NPRP) grant 9-350-3-074 (to RA).

Address correspondence to: Reza Abdi, Transplantation Research Center, Brigham and Women's Hospital, 221 Longwood Avenue, Boston, Massachusetts 02116, USA. Phone: 617.732.5259, Email: rabdi@rics.bwh.harvard.edu.
1. Legendre C, Canaud G, Martinez F. Factors influencing long-term outcome after kidney transplantation. Transpl Int. 2014;27(1):19-27.

2. Bai Y, et al. L-selectin-dependent lymphoid occupancy is required to induce alloantigen-specific tolerance. JImmunol. 2002;168(4):1579-1589.

3. Billingham RE, Brent L, Medawar PB. 'Actively acquired tolerance' of foreign cells. 1953. JImmunol. 2010;184(1):5-8.

4. Raut A, Huryn J, Pollack A, Zlotolow I. Unusual gingival presentation of post-transplantation lymphoproliferative disorder: a case report and review of the literature. Oral Surg Oral Med Oral Pathol Oral Radiol Endod. 2000;90(4):436-441.

5. Lakkis FG. Transplantation tolerance: a journey from ignorance to memory. Nephrol Dial Transplant. 2003;18(10):1979-1982.
6. Brinkman CC, et al. Anatomy of tolerance. Curr Opin Organ Transplant. 2013;18(4):393-401.

7. Burrell BE, et al. Tolerance and lymphoid organ structure and function. Front Immunol. 2011;2:64.

8. Golshayan D, Jiang S, Tsang J, Garin MI, Mottet C, Lechler RI. In vitro-expanded donor alloantigenspecific $\mathrm{CD} 4{ }^{+} \mathrm{CD} 25^{+}$regulatory $\mathrm{T}$ cells promote experimental transplantation tolerance. Blood. 2007;109(2):827-835.

9. Tang $\mathrm{Q}$, et al. Visualizing regulatory $\mathrm{T}$ cell contro of autoimmune responses in nonobese diabetic mice. Nat Immunol. 2006;7(1):83-92.

10. Warren KJ, Iwami D, Harris DG, Bromberg JS, Burrell BE. Laminins affect $T$ cell trafficking and allograft fate. JClin Invest. 2014;124(5):2204-2218.

11. Bahmani B, Vohra I, Kamaly N, Abdi R. Active targeted delivery of immune therapeutics to lymph nodes. Curr Opin Organ Transplant. 2018;23(1):8-14.

12. Yu B, Tai HC, Xue W, Lee LJ, Lee RJ. Receptortargeted nanocarriers for therapeutic delivery to cancer. Mol Membr Biol. 2010;27(7):286-298.

13. Kolhatkar R, Lote A, Khambati H. Active tumor targeting of nanomaterials using folic acid, transferrin and integrin receptors. Curr Drug Discov Technol. 2011;8(3):197-206.

14. Peer D, Karp JM, Hong S, Farokhzad OC, Margalit R, Langer R. Nanocarriers as an emerging platform for cancer therapy. Nat Nanotechnol. 2007;2(12):751-760.

15. Farokhzad OC, et al. Targeted nanoparticleaptamer bioconjugates for cancer chemotherapy in vivo. Proc Natl Acad Sci US A. 
2006;103(16):6315-6320.

16. Lammers T, Hennink WE, Storm G. Tumourtargeted nanomedicines: principles and practice. Br J Cancer. 2008;99(3):392-397.

17. von Andrian UH, Mackay CR. T-cell function and migration. Two sides of the same coin. $N$ Engl J Med. 2000;343(14):1020-1034.

18. Uchimura K, et al. A major class of L-selectin ligands is eliminated in mice deficient in two sulfotransferases expressed in high endothelial venules. Nat Immunol. 2005;6(11):1105-1113.

19. Uchimura K, Rosen SD. Sulfated L-selectin ligands as a therapeutic target in chronic inflammation. Trends Immunol. 2006;27(12):559-565.

20. Choi HS, et al. Renal clearance of quantum dots. Nat Biotechnol. 2007;25(10):1165-1170.

21. Contado C, Vighi E, Dalpiaz A, Leo E. Influence of secondary preparative parameters and aging effects on PLGA particle size distribution: a sedimentation field flow fractionation investigation. Anal Bioanal Chem. 2013;405(2-3):703-711.

22. Li SD, Huang L. Pharmacokinetics and biodistribution of nanoparticles. Mol Pharm. 2008;5(4):496-504.

23. Kawashima H, et al. N-acetylglucosamine6-O-sulfotransferases 1 and 2 cooperatively control lymphocyte homing through L-selectin ligand biosynthesis in high endothelial venules. Nat Immunol. 2005;6(11):1096-1104.

24. Richards DA, Maruani A, Chudasama V. Antibody fragments as nanoparticle targeting ligands: a step in the right direction. Chem Sci. 2017;8(1):63-77.

25. Goswami S, Wang W, Arakawa T, Ohtake S. Developments and challenges for $\mathrm{mAb}$-based therapeutics. Antibodies. 2013;2(3):452-500.

26. Halloran PF. Immunosuppressive drugs for kidney transplantation. N Engl JMed. 2004;351(26):2715-2729.

27. Kirk AD, Mannon RB, Swanson SJ, Hale DA. Strategies for minimizing immunosuppression in kidney transplantation. Transpl Int. 2005;18(1):2-14.

28. Nashan B. Antibody induction therapy in renal transplant patients receiving calcineurin-inhibitor immunosuppressive regimens: a comparative review. BioDrugs. 2005;19(1):39-46.

29. Andreoni KA, Brayman KL, Guidinger MK, Sommers CM, Sung RS. Kidney and pancreas transplantation in the United States, 1996-2005. Am J Transplant. 2007;7(5 pt 2):1359-1375.

30. Chatenoud L, Bluestone JA. CD3-specific antibodies: a portal to the treatment of autoimmunity. Nat Rev Immunol. 2007;7(8):622-632.

31. Cosimi AB, et al. Use of monoclonal antibodies to $\mathrm{T}$-cell subsets for immunologic monitoring and treatment in recipients of renal allografts. $N$ Engl JMed. 1981;305(6):308-314.

32. Herold KC, et al. Anti-CD3 monoclonal antibody in new-onset type 1 diabetes mellitus. $N$ Engl J Med. 2002;346(22):1692-1698.

33. Chatenoud L. CD3-specific antibody-induced active tolerance: from bench to bedside. Nat Rev Immunol. 2003;3(2):123-132.

34. Bisikirska B, Colgan J, Luban J, Bluestone JA, Herold KC. TCR stimulation with modified anti-CD3 $\mathrm{mAb}$ expands $\mathrm{CD} 8^{+} \mathrm{T}$ cell population and induces $\mathrm{CD} 8^{+} \mathrm{CD} 25^{+}$Tregs. JClin Invest. 2005;115(10):2904-2913.
35. Billingham ME, et al. A working formulation for the standardization of nomenclature in the diagnosis of heart and lung rejection: Heart Rejection Study Group. The International Society for Heart Transplantation. J Heart Transplant. 1990;9(6):587-593.

36. Stewart S, et al. Revision of the 1990 working formulation for the standardization of nomenclature in the diagnosis of heart rejection. J Heart Lung Transplant. 2005;24(11):1710-1720.

37. Sgro C. Side-effects of a monoclonal antibody, muromonab CD3/orthoclone OKT3: bibliographic review. Toxicology. 1995;105(1):23-29.

38. Ibrahim Z, et al. A modified heterotopic swine hind limb transplant model for translational vascularized composite allotransplantation (VCA) research. J Vis Exp. 2013;(80):50475.

39. Hunter Z, et al. A biodegradable nanoparticle platform for the induction of antigen-specific immune tolerance for treatment of autoimmune disease. ACS Nano. 2014;8(3):2148-2160.

40. Getts DR, Shea LD, Miller SD, King NJ. Harnessing nanoparticles for immune modulation. Trends Immunol. 2015;36(7):419-427.

41. Hlavaty KA, Luo X, Shea LD, Miller SD. Cellular and molecular targeting for nanotherapeutics in transplantation tolerance. Clin Immunol. 2015;160(1):14-23.

42. Reddy ST, Swartz MA, Hubbell JA. Targeting dendritic cells with biomaterials: developing the next generation of vaccines. Trends Immunol. 2006;27(12):573-579.

43. Dane KY, et al. Nano-sized drug-loaded micelles deliver payload to lymph node immune cells and prolong allograft survival. J Control Release. 2011;156(2):154-160.

44. Hubbell JA, Thomas SN, Swartz MA. Materials engineering for immunomodulation. Nature. 2009;462(7272):449-460.

45. Reddy ST, et al. Exploiting lymphatic transport and complement activation in nanoparticle vaccines. Nat Biotechnol. 2007;25(10):1159-1164.

46. Rincon-Restrepo M, et al. Vaccine nanocarriers: coupling intracellular pathways and cellular biodistribution to control CD4 vs CD8 T cell responses. Biomaterials. 2017;132:48-58.

47. Liu H, et al. Structure-based programming of lymph-node targeting in molecular vaccines. Nature. 2014;507(7493):519-522.

48. Jewell CM, López SC, Irvine DJ. In situ engineering of the lymph node microenvironment via intranodal injection of adjuvant-releasing polymer particles. Proc Natl Acad Sci U S A. 2011;108(38):15745-15750.

49. Blanco E, Shen H, Ferrari M. Principles of nanoparticle design for overcoming biological barriers to drug delivery. Nat Biotechnol. 2015;33(9):941-951.

50. Azzi J, et al. Targeted delivery of immunomodulators to lymph nodes. Cell Rep. 2016;15(6):1202-1213.

51. Burns JA, Butler JC, Moran J, Whitesides GM. Selective reduction of disulfides by tris (2-carboxyethyl) phosphine.JOrg Chem. 1991;56(8):2648-2650.

52. Brandtzaeg P, Prydz H. Direct evidence for an integrated function of J chain and secretory component in epithelial transport of immuno- globulins. Nature. 1984;311(5981):71-73.

53. Sørensen V, Rasmussen IB, Sundvold V, Michaelsen TE, Sandlie I. Structural requirements for incorporation of J chain into human IgM and IgA. Int Immunol. 2000;12(1):19-27.

54. Kikuchi H, Goto Y, Hamaguchi K. Reduction of the buried intrachain disulfide bond of the constant fragment of the immunoglobulin light chain: global unfolding under physiological conditions. Biochemistry. 1986;25(8):2009-2013.

55. Lefranc MP, et al. IMGT unique numbering for immunoglobulin and $\mathrm{T}$ cell receptor constant domains and Ig superfamily C-like domains. Dev Comp Immunol. 2005;29(3):185-203.

56. Amzel LM, Poljak RJ. Three-dimensional structure of immunoglobulins. Annu Rev Biochem. 1979;48:961-997.

57. Liu H, May K. Disulfide bond structures of IgG molecules: structural variations, chemical modifications and possible impacts to stability and biological function. MAbs. 2012;4(1):17-23.

58. Kownatzki E. Disulfide bonds of human IgM: differential sensitivity to reductive cleavage. Scand J Immunol. 1973;2(4):433-437.

59. Zhang X, et al. 3D structural fluctuation of IgG1 antibody revealed by individual particle electron tomography. Sci Rep. 2015;5:9803.

60. Bajénoff M, Granjeaud S, Guerder S. The strategy of $\mathrm{T}$ cell antigen-presenting cell encounter in antigen-draining lymph nodes revealed by imaging of initial T cell activation. J Exp Med. 2003;198(5):715-724.

61. Wagh A, Law B. Methods for conjugating antibodies to nanocarriers. Methods Mol Biol. 2013;1045:249-266.

62. Jamil B, Nicholls K, Becker GJ, Walker RG. Impact of acute rejection therapy on infections and malignancies in renal transplant recipients. Transplantation. 1999;68(10):1597-1603.

63. Bustami RT, et al. Immunosuppression and the risk of post-transplant malignancy among cadaveric first kidney transplant recipients. Am J Transplant. 2004;4(1):87-93.

64. Stratta $P$, et al. Malignancy after kidney transplantation: results of 400 patients from a single center. Clin Transplant. 2008;22(4):424-427.

65. Cantarovich D, et al. Corticosteroid avoidance in adult kidney transplant recipients under rabbit anti-T-lymphocyte globulin, mycophenolate mofetil and delayed cyclosporine microemulsion introduction. Transpl Int. 2010;23(3):313-324.

66. Goto R, You S, Zaitsu M, Chatenoud L, Wood KJ. Delayed anti-CD3 therapy results in depletion of alloreactive T cells and the dominance of Foxp3+ CD4+ graft infiltrating cells. Am J Transplant. 2013;13(7):1655-1664.

67. Kendal AR, et al. Sustained suppression by Foxp $3^{+}$regulatory $\mathrm{T}$ cells is vital for infectious transplantation tolerance. J Exp Med. 2011;208(10):2043-2053.

68. Penaranda C, Tang Q, Bluestone JA. Anti-CD3 therapy promotes tolerance by selectively depleting pathogenic cells while preserving regulatory T cells. J Immunol. 2011;187(4):2015-2022.

69. Alegre ML, et al. Cytokine release syndrome induced by the 145-2C11 anti-CD3 monoclonal antibody in mice: prevention by high 
doses of methylprednisolone. JImmunol. 1991;146(4):1184-1191.

70. Ferran C, et al. Cytokine-related syndrome following injection of anti-CD3 monoclonal antibody: further evidence for transient in vivo $\mathrm{T}$ cell activation. Eur J Immunol. 1990;20(3):509-515.

71. Hirsch R, Eckhaus M, Auchincloss H, Sachs $\mathrm{DH}$, Bluestone JA. Effects of in vivo adminis- tration of anti-T3 monoclonal antibody on $\mathrm{T}$ cell function in mice. I. Immunosuppression of transplantation responses. J Immunol. 1988;140(11):3766-3772.

72. Michie HR, et al. Detection of circulating tumor necrosis factor after endotoxin administration. N Engl J Med. 1988;318(23):1481-1486.

73. Berg EL, Robinson MK, Warnock RA, Butcher EC. The human peripheral lymph node vascular addressin is a ligand for LECAM-1, the peripheral lymph node homing receptor. JCell Biol. 1991;114(2):343-349.

74. Corry RJ, Winn HJ, Russell PS. Heart transplantation in congenic strains of mice. Transplant Proc. 1973;5(1):733-735.

75. Ionac M. One technique, two approaches, and results: thoracic duct cannulation in small laboratory animals. Microsurgery. 2003;23(3):239-245. 